\title{
Mutations in multiple components of the nuclear pore complex cause nephrotic syndrome
}

Daniela A. Braun,,$^{1,2}$ Svjetlana Lovric,, David Schapiro, ${ }^{1}$ Ronen Schneider, ${ }^{1}$ Jonathan Marquez, ${ }^{3}$ Maria Asif, $4,5,6$

Muhammad Sajid Hussain, ${ }^{4,5,7}$ Ankana Daga, ${ }^{1}$ Eugen Widmeier, Jia Rao ${ }^{8,9}$ Shazia Ashraf, ${ }^{1}$ Weizhen Tan, ${ }^{1}$ C. Patrick Lusk, ${ }^{10}$ Amy Kolb, ${ }^{1}$ Tilman Jobst-Schwan, ${ }^{1}$ Johanna Magdalena Schmidt, ${ }^{1}$ Charlotte A. Hoogstraten, ${ }^{1}$ Kaitlyn Eddy, ${ }^{1}$ Thomas M. Kitzler, Shirlee Shril, ${ }^{1}$ Abubakar Moawia, ${ }^{4,5,6}$ Kathrin Schrage, ${ }^{5}$ Arwa Ishaq A. Khayyat, ${ }^{5,11}$ Jennifer A. Lawson, ${ }^{1}$ Heon Yung Gee, ${ }^{1}$ jillian K. Warejko, ${ }^{1}$ Tobias Hermle, ${ }^{1}$ Amar J. Majmundar, ${ }^{1}$ Hannah Hugo, ${ }^{1}$ Birgit Budde, ${ }^{4}$ Susanne Motameny, ${ }^{4}$ Janine Altmüller, ${ }^{4,7112}$ Angelika Anna Noegel, ${ }^{5,7,13}$ Hanan M. Fathy, ${ }^{14}$ Daniel P. Gale, ${ }^{15}$ Syeda Seema Waseem,,${ }^{4,5,6}$ Ayaz Khan, ${ }^{6}$ Larissa Kerecuk, ${ }^{16}$ Seema Hashmi, ${ }^{17}$ Nilufar Mohebbi, ${ }^{18}$ Robert Ettenger, ${ }^{19}$ Erkin Serdaroğlu, ${ }^{20}$ Khalid A. Alhasan, ${ }^{21}$ Mais Hashem, $, 22,23,24$ Sara Goncalves, ${ }^{25,26}$ Gema Ariceta, ${ }^{27}$ Mercedes Ubetagoyena, ${ }^{28}$ Wolfram Antonin, ${ }^{29}$ Shahid Mahmood Baig, ${ }^{6}$ Fowzan S. Alkuraya, ${ }^{22,23,24}$ Qian Shen, ${ }^{8,9}$ Hong Xu, ${ }^{8,9}$ Corinne Antignac, ${ }^{25,26,30}$ Richard P. Lifton, ${ }^{31,32}$ Shrikant Mane, ${ }^{31}$ Peter Nürnberg, ${ }^{4,7,3}$ Mustafa K. Khokha, ${ }^{3}$ and Friedhelm Hildebrandt ${ }^{1}$

\begin{abstract}
'Department of Medicine, Boston Children's Hospital, Harvard Medical School, Boston, Massachusetts, USA. ² Department of Internal Medicine D, University Hospital of Münster, Münster, Germany. ${ }^{3}$ Pediatric Genomics Discovery Program, Department of Pediatrics and Genetics, Yale University School of Medicine, New Haven, Connecticut, USA. ${ }^{4}$ Cologne Center for Cenomics, University of Cologne, Cologne, Germany. 5Institute of Biochemistry I, Medical Faculty, University of Cologne, Cologne, Germany. ${ }^{\circ}$ Human Molecular Cenetics Laboratory, Health Biotechnology Division, National Institute for Biotechnology and Genetic Engineering, Pakistan Institute of Engineering and Applied Sciences, Faisalabad, Pakistan. 'Center for Molecular Medicine Cologne, University of Cologne, Cologne, Cermany. ${ }^{8}$ Department of Nephrology, Children's Hospital of Fudan University, Shanghai, China. ${ }^{9}$ Shanghai Kidney Development and Pediatric Kidney Disease Research Center, Shanghai, China. ${ }^{10}$ Department of Cell Biology, Yale University School of Medicine, New Haven, Connecticut, USA. "Biochemistry Department, King Saud University, Riyadh, Saudi Arabia. ${ }^{12}$ Institute of Human Genetics, University of Cologne, Cologne, Germany. ${ }^{13}$ Cologne Excellence Cluster on Cellular Stress Responses in Aging-Associated Diseases (CECAD), University of Cologne, Cologne, Germany. ${ }^{14}$ Pediatric Nephrology Unit, Alexandria Faculty of Medicine, University of Alexandria, Alexandria, Egypt. ${ }^{15}$ Centre for Nephrology, University College London, Royal Free Hospital, London, United Kingdom. ${ }^{16 B i r m i n g h a m ~ C h i l d r e n ' s ~ H o s p i t a l ~ N H S ~ F o u n d a t i o n ~ T r u s t, ~ B i r m i n g h a m, ~ U n i t e d ~ K i n g d o m . ~}{ }^{17}$ Department of Pediatric Nephrology, Sindh Institute of Urology and Transplantation, Karachi, Pakistan. ${ }^{18}$ Division of Nephrology, University Hospital Zurich, Zurich, Switzerland. ${ }^{19}$ Department of Pediatrics, University of California, Los Angeles, California. ${ }^{20}$ Department of Pediatric Nephrology, Dr. Behçet Uz Children's Hospital, Izmir, Turkey. ${ }^{21 P e d i a t r i c ~ D e p a r t m e n t, ~ C o l l e g e ~ o f ~ M e d i c i n e, ~ K i n g ~ S a u d ~ U n i v e r s i t y ~ a n d ~ K i n g ~ K h a l i d ~ U n i v e r s i t y ~ H o s p i t a l, ~}$ Riyadh, Saudi Arabia. ${ }^{22}$ Department of Cenetics, King Faisal Specialist Hospital and Research Center, Riyadh, Saudi Arabia. ${ }^{23}$ Department of Anatomy and Cell Biology, College of Medicine, Alfaisal University, Riyadh, Saudi Arabia. ${ }^{24}$ Saudi Human Genome Program, King Abdulaziz City for Science and Technology, Riyadh, Saudi Arabia. ${ }^{25}$ Laboratory of Hereditary Kidney Diseases, INSERM UMR1163, Imagine Institute, Paris, France. ${ }^{26}$ Université Paris Descartes-Sorbonne Paris Cité, Imagine Institute, Paris, France. ${ }^{27}$ Universitat Autonoma de Barcelona, Hospital Universitari Vall d'Hebron, Pediatric Nephrology, Barcelona, Spain. ${ }^{28}$ Hospital Universitario Donostia, Pediatric Nephrology, Donostia-San Sebastian, Spain. ${ }^{29}$ nstitute of Biochemistry and Molecular Cell Biology, Medical School, RWTH Aachen University, 52074 Aachen, Germany. ${ }^{30}$ Department of Genetics, Necker Hospital, Assistance Publique-Hôpitaux de Paris, Paris, France. ${ }^{31}$ Department of Genetics, Yale University School of Medicine, New Haven, Connecticut, USA. ${ }^{32}$ Laboratory of Human Genetics and Genomics, The Rockefeller University, New York, New York, USA.
\end{abstract}

\begin{abstract}
Steroid-resistant nephrotic syndrome (SRNS) almost invariably progresses to end-stage renal disease. Although more than $\mathbf{5 0}$ monogenic causes of SRNS have been described, a large proportion of SRNS remains unexplained. Recently, it was discovered that mutations of NUP93 and NUP205, encoding 2 proteins of the inner ring subunit of the nuclear pore complex (NPC), cause SRNS. Here, we describe mutations in genes encoding 4 components of the outer rings of the NPC, namely NUP107, NUP85, NUP133, and NUP160, in 13 families with SRNS. Using coimmunoprecipitation experiments, we showed that certain pathogenic alleles weakened the interaction between neighboring NPC subunits. We demonstrated that morpholino knockdown of nup107, nup85, or nup133 in Xenopus disrupted glomerulogenesis. Re-expression of WT mRNA, but not of mRNA reflecting mutations from SRNS patients, mitigated this phenotype. We furthermore found that CRISPR/Cas9 knockout of NUP107, NUP85, or NUP133 in podocytes activated Cdc42, an important effector of SRNS pathogenesis. CRISPR/ Cas9 knockout of nup107 or nup85 in zebrafish caused developmental anomalies and early lethality. In contrast, an in-frame mutation of nup107 did not affect survival, thus mimicking the allelic effects seen in humans. In conclusion, we discovered here that mutations in 4 genes encoding components of the outer ring subunits of the NPC cause SRNS and thereby provide further evidence that specific hypomorphic mutations in these essential genes cause a distinct, organ-specific phenotype.
\end{abstract}

Conflict of interest: FH is a cofounder of Goldfinch Bio Inc. and receives royalties from Claritas Genomics.

Submitted: November 16, 2017; Accepted: July 24, 2018.

Reference information: J Clin Invest. 2018;128(10):4313-4328.

https://doi.org/10.1172/JCI98688.

\section{Introduction}

Steroid-resistant nephrotic syndrome (SRNS), a disease of the renal glomerular filter, is characterized by proteinuria, edema, and hypoalbuminemia. In contrast to other forms of nephrotic syndrome, SRNS does not respond to drug treatment and 
inevitably progresses to end-stage renal disease (ESRD), thus requiring dialysis or renal transplantation for survival (1). It constitutes the second most frequent cause of ESRD in the first 3 decades of life (2). In SRNS, renal histology reveals focal segmental glomerulosclerosis or diffuse mesangial sclerosis, which indicate irreversible damage to the glomerulus. Mutations in over 50 genes have been discovered to cause monogenic SRNS $(3,4)$. These genetic findings have revealed podocytes, specialized epithelial cells of the glomerular filter, as the critical site of injury in SRNS $(5,6)$. Furthermore, identification of disease genes has implicated multiple signaling pathways in the molecular pathogenesis of SRNS (3). Our group and others have recently demonstrated that in about $30 \%$ of patients with SRNS a causative single-gene mutation can be identified (7-9).

The nuclear pore complex (NPC) is a large (100 MDa) macromolecular assembly that spans the nuclear envelope and forms a selective barrier between the cytoplasm and the nucleoplasm (10). The central channel of the NPC is filled with intrinsically disordered proteins, which are rich in phenylalanine-glycine (FG) amino acid residues. These FG-rich nucleoporins (NUPs) establish a size-selective diffusion barrier to macromolecules greater than approximately $40 \mathrm{kDa}$ while providing binding sites for nuclear transport receptors (karyopherins, importins, and exportins) that ferry signal-bearing cargo across the NPC (10). The central channel is supported by a structural scaffold built from the inner and outer ring complexes, which are formed by repeating modular units of the NUP93 subcomplex and the NUP107-160 complex, or "Y complex," respectively $(11,12)$. The Y complex is the major subunit of the outer rings of the NPC. The atomic structures of the major domains of all 7 members of the Y complex (either alone or in complex), including NUPs $43,85,96,107,133$, and 160 , have been solved and modeled into electron cryomicroscopic maps of the human and Xenopus NPC (10).

Interestingly, while NUPs are best understood in their roles at the NPC, they also participate in additional functions in other subcellular locations (13-15). Studies in vertebrate model organisms additionally suggest that NUPs may have important regulatory functions during development and for tissue-specific progenitor cell maturation $(16,17)$. Genetic depletion of NUP transcripts caused severe developmental defects in zebrafish (18), mice (16), and frogs (15). Interestingly, some NUPencoding genes show differential expression in different tissues and in different developmental stages $(15,19)$. NUPs studied in this article (i.e., nup107, nup85, nup133, and nup160) were shown to be expressed in both the rostral portion of the embryo (relevant to CNS development) and the intermediate mesoderm (important for pronephric development) (20).

Recently, we identified mutations in NUP93 and NUP205, encoding 2 components of the inner ring subunit of the NPC, in patients with nonsyndromic SRNS (21). Intriguingly, patients with mutations in NUP107, encoding a component of the Y complex, also developed SRNS (22-24). These observations suggest that a specific pathogenic link between SRNS development and alterations in different NUPs exists.

Performing whole exome sequencing and targeted exon sequencing, we here identified mutations in 4 genes encoding components of the outer ring subunits of the NPC, namely NUP107,
NUP85, NUP133, and NUP160, in 29 individuals of 13 families with SRNS. Furthermore, we detected a homozygous mutation in NUP37 in a family with primary microcephaly, a phenotype that was also present in some families with NUP107 mutations. We use different in vivo and in vitro models to elucidate parts of the related pathogenesis.

\section{Results}

Hypomorphic mutations in 4 different genes encoding components of the outer ring subunits of the NPC cause nephrotic syndrome. To identify additional genes that cause SRNS if mutated, we performed whole exome sequencing (25) in 160 families with SRNS. After excluding mutations in known SRNS genes, we used homozygosity mapping (26) in consanguineous families to identify potentially novel candidate loci for SRNS. This approach yielded a homozygous missense mutation (c.303G $>$ A, p.Met101Ile) in the gene NUP1O7 (NM_020401.3) (Table 1 and Figure 1, B-D) in consanguineous family A4649 with 3 affected siblings with SRNS and microcephaly. In 4 additional consanguineous families with a similar phenotype (B1426, A802, FA, PN-1), the NUP107 locus was positioned within a homozygous peak region, and we identified the same mutation in NUP1O7 (Figure 1, B-D, Supplemental Table 1, Supplemental Figure 1A, and Supplemental Figures 2 and 3; supplemental material available online with this article; https:// doi.org/10.1172/JCI98688DS1). The p.Met101Ile mutation represents a South Asian founder allele that was reported previously in patients with the combined phenotype of SRNS and primary microcephaly $(23,27)$. Prior studies demonstrated that the p.Met101Ile mutation of NUP107 results in aberrant splicing and causes a reduction in the NUP107 protein level (23). NUP107 is an essential protein for NPC assembly $(28,29)$. In consanguineous family F797 with nonsyndromic SRNS, we found a homozygous missense mutation (c.2922T>G, p.Ser974Arg) in the gene NUP133 (NM_018230.2), which was located within a homozygous peak region on chromosome 1 (Figure $1, \mathrm{H}-\mathrm{J}$ ).

Because NUP133 and NUP107 encode 2 interacting components of the outer ring subunits of the NPC, we hypothesized that alterations in other nuclear pore proteins may also cause monogenic SRNS. We therefore performed targeted sequencing of all exons of 19 genes that encode other NUPs in a worldwide cohort of 2,164 families with SRNS using a multiplex PCR-based high-throughput sequencing strategy $(7,30,31)$ or using whole exome sequencing. As a result, we detected a homozygous missense mutation of NUP107 (c.2666A>G, p.Tyr889Cys) in family A3825 and 2 compound heterozygous alleles (c.1021dup, p.Glu341Glyfs ${ }^{*}$, and c.2129_2131delAAG, p.Glu710del) of NUP107 in family A1830. None of these alleles were reported previously (Table 1 and Figure 1, A-D). All 5 families who carried the p.Met101Ile allele and family A1830 displayed the combined phenotype of SRNS with microcephaly and intellectual disability. In contrast, family A3825 and 9 previously reported families with NUP107 alleles other than p.Met101Ile did not have a neurodevelopmental phenotype $(22,24)$. We speculate that this allelism may be explained by the fact that only the homozygous p.Met101Ile allele resulted in a protein-truncating mutation (23), while all other families carried at least 1 recessive missense allele. This observation suggests that, as seen in other monogenic dis- 


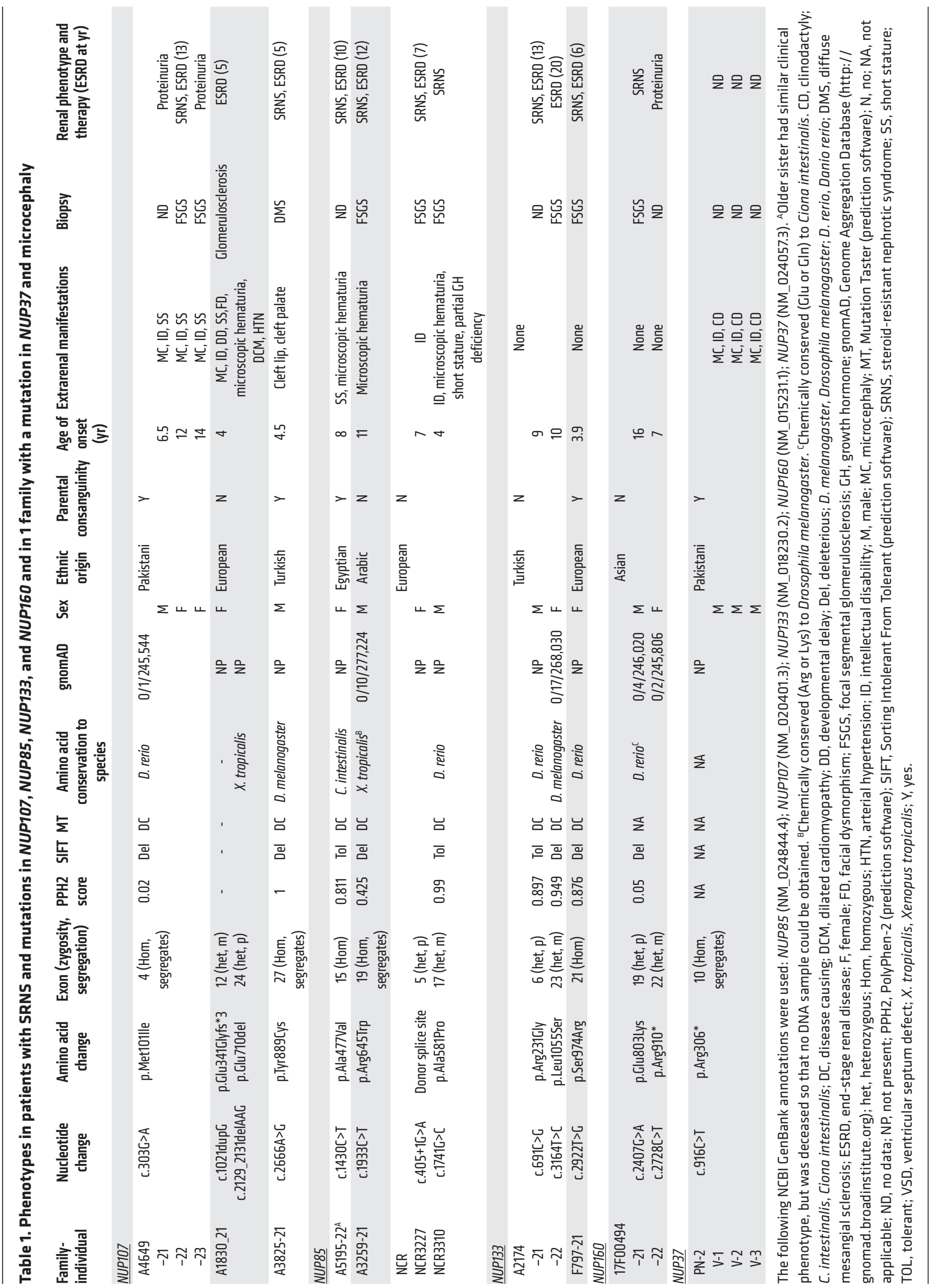


A

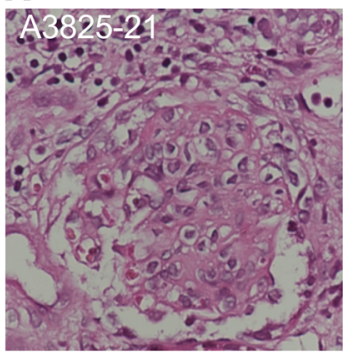

C

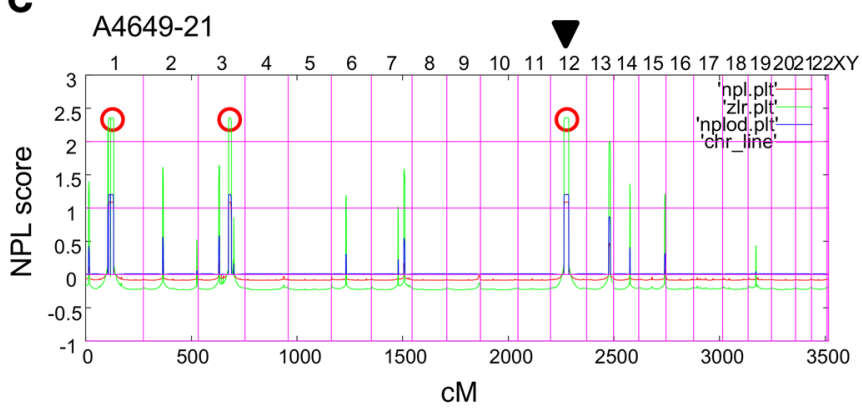

E

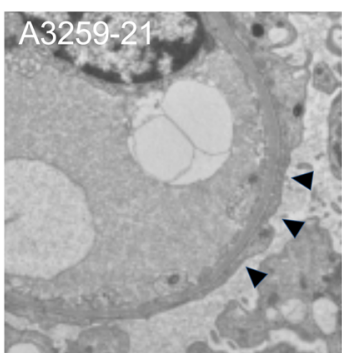

H

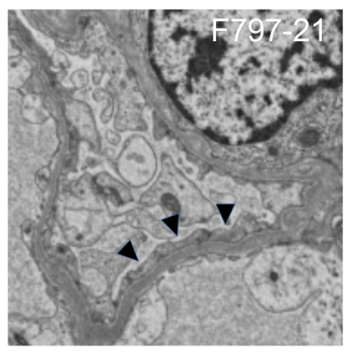

J

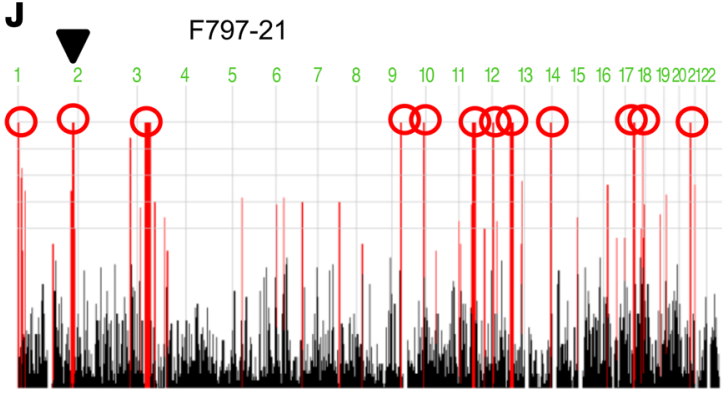

\section{$\mathbf{F}$}

NUP85 (NM_024844.4)

\section{I}

NUP133 (NM_018230.2)

ATG

$3471 \mathrm{bp}$
B

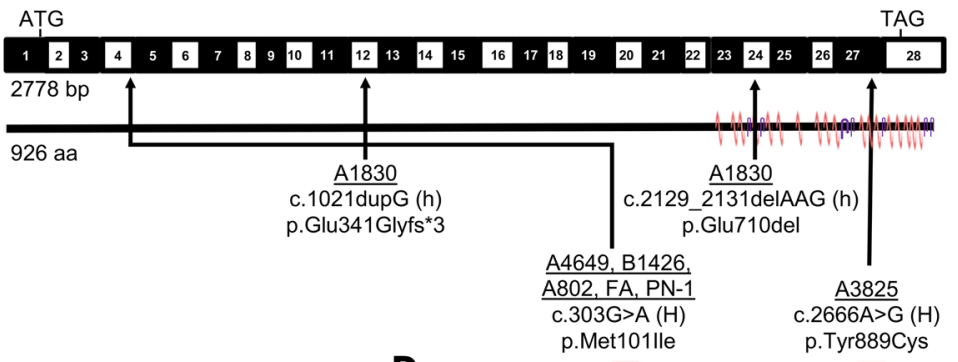

D

H. sapiens

M. musculus

G. gallus

$X$. tropicalis

D. rerio

C. intestinalis

D. melanogast $\nabla$

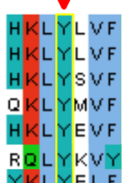

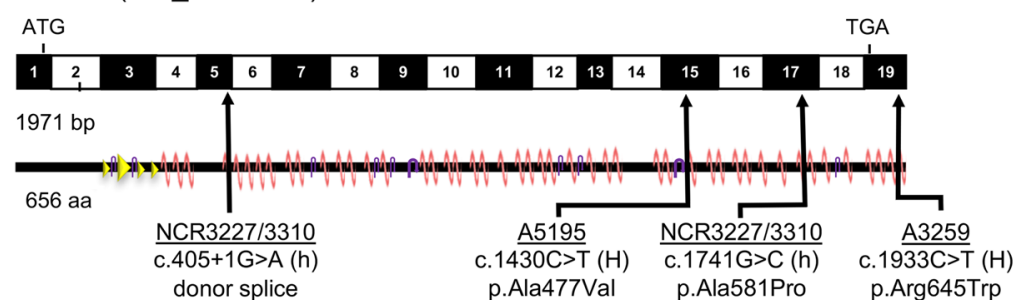
G H. sapiens M. musculus
G. gallus
$X$. tropicalis
$D$. rerio
C. intestinalis
D. melanogaster
C. elegans
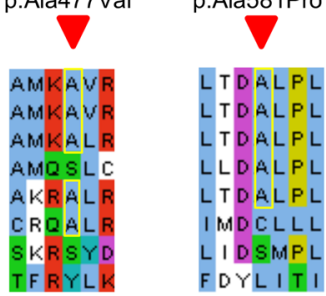

$\nabla$

NLARA I I

NLARAI I

NLARVIV

NLARAIV

NLATA I V

NLA互A I V

NLARAL I

SIAKG I A

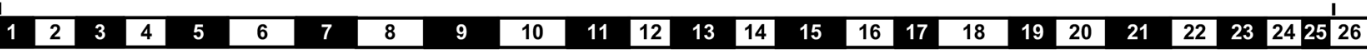

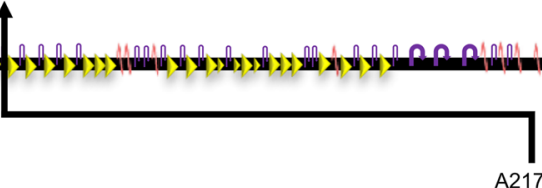

$\mathbf{K}$

H. sapiens

M. musculus

G. gallus

X. tropicalis

D. rerio

C. intestinalis

D. melanogaster

C. elegans c. $\frac{\mathrm{A} 2174}{\mathrm{C}>\mathrm{G}}$

p.Arg231Gly

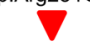

QL I RL - I

Q L VRL - I

QLVRL - I

HLVRL - T

HLLEL - S

AVYQL - Q

NLVFLRV

T VYF L - N

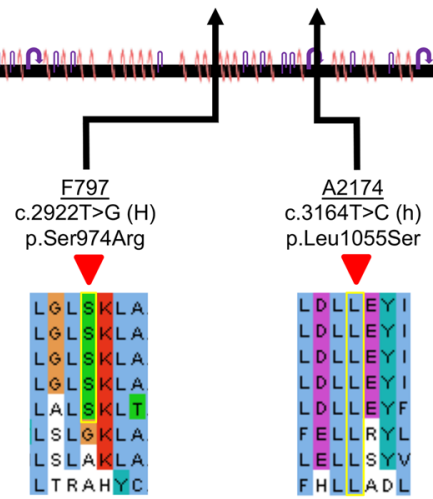


Figure 1. Homozygosity mapping and whole exome sequencing identify recessive mutations of NUP107, NUP85, and NUP133 in 12 families with steroid-resistant nephrotic syndrome. (A) Renal histology of individual A3825-21 (NUP107 mutation) shows diffuse mesangial sclerosis on light microscopy. (B, F, and I) Exon structure of human cDNAs. Positions of start codons and of stop codon are indicated. For protein domain structures, $\alpha$-helices are depicted as red zigzag lines and $\beta$-turns as purple arrows. Arrows indicate positions of pathogenic mutations detected in families with SRNS. H, homozygous; h, heterozygous. (B) Exon structure, protein domain structure, and human mutations of NUP107. (C) Homozygosity mapping identifies 3 recessive candidate loci (red circles) in patient A4649-21. Nonparametric lod (NPL) scores and SNP positions (Affymetrix 250K Styl array) are plotted on human chromosomes concatenated from $\mathrm{p}$-ter (left) to q-ter (right). Genetic distance is given in centimorgans (cM). Whole exome sequencing identifies a homozygous mutation of NUP107 (p.Met101lle) that is positioned within the maximum NPL peak on chromosome 12 (arrowhead). (D, G, and K) Evolutionary conservation of amino acid residues that are altered in patients with SRNS. (D) Altered amino acid residues of NUP107 (p.Met101lle, p.Tyr889Cys). (E) Renal histology of A3259-21 (NUP85 mutation) showing podocyte foot process effacement on transmission electron microscopy (TEM) (arrowheads). (F) Exon structure, protein domain structure, and human mutations of NUP85. (G) Altered amino acid residues of NUP85 (p.Ala477Val, p.Ala581Pro, p.Arg645Trp). (H) Renal histology of individual F797-21 (NUP133 mutation) shows podocyte foot process effacement on TEM (arrowheads). (I) Exon structure, protein domain structure, and human mutations of NUP133. (J) Homozygosity mapping in individual F797-21 identifies regions of homozygosity as recessive candidate loci. Within the maximum NPL peak on chromosome 1 (arrowhead), we identified a homozygous mutation in NUP133 (p.Ser974Arg). (K) Altered amino acid residues of NUP133 (p.Arg231Gly, p.Ser974Arg, p.Leu1055Ser).

eases (32), severe mutations in NUP genes cause syndromic phenotypes, while milder mutations give rise to distinct organspecific phenotypes. Besides microcephaly, 2 families (B1426 and A3825) showed additional skeletal/facial phenotypes (in particular arachnodactyly, high arched palate, and cleft palate/ lip), and 1 family (A802) had congenital heart disease (Table 1 and Supplemental Table 1). The targeted screening additionally identified 2 segregating, compound heterozygous mutations of NUP133 (c.691C>G, p.Arg231Gly, and c.3164T>C, p.Leu1055Ser, NM 018230.2) in family A2174 with nonsyndromic SRNS (Figure 1, I and K; Supplemental Figure 1C; and Table 1).

High-throughput exon sequencing furthermore yielded 3 mutant alleles of NUP85 (NM_024844.4), encoding a different component of the outer rings of the NPC, in 4 individuals of 3 unrelated families with SRNS (families A5195, A3259, and NCR3227/3310) (Figure 1, E-G; Supplemental Figure 1B; and Table 1). Two mutations were homozygous missense mutations (c.1430C $>$ T, p.Ala477Val, and c.1933C $>$ T, p.Arg645Trp). One family (NCR3227/3310) carried 2 compound heterozygous alleles $(\mathrm{c} .405+1 \mathrm{G}>\mathrm{A}$ and c. $1741 \mathrm{G}>\mathrm{C}$, p.Ala581Pro), which segregated from the maternal and the paternal side, respectively (Table 1). All families with NUP85 mutations had SRNS and microscopic hematuria, a finding that can be associated with SRNS. Family NCR3227/3310 additionally displayed intellectual disability, but showed no structural brain defects. Interestingly, in several families with NUP107 or NUP85 mutations, short stature was noted as a clinical feature, and family NCR3227/3310 had partial growth hormone deficiency.

Using the list of 19 candidate genes for targeted evaluation of whole exome sequencing data in a non-consanguineous Chi- nese family, we discovered 2 compound heterozygous mutations (c.2407G $>$ A, p.Glu803Lys, and c.2728C $>$ T, p.Arg910*) in the gene NUP160 (NM_015231.1; Table 1 and Supplemental Figure 4). NUP160 encodes a direct interaction partner of NUP85 within the Y complex (Figure 2A). The older sibling presented at age 16 years with nephrotic syndrome that was resistant to therapy with steroids or other immunosuppressive drugs. His biopsy showed focal segmental glomerulosclerosis (FSGS), and his renal function was impaired (chronic kidney disease stage 3). The younger sister had proteinuria that first presented at age 7 years. Neither of the siblings displayed extrarenal symptoms (Table 1).

Twenty-five individuals of 13 families with recessive mutations of NUP107, NUP85, NUP133, or NUP160 had SRNS or proteinuria that manifested in childhood or adolescence (Table 1 and Supplemental Table 1). In 13 of these 25 patients, the disease progressed to ESRD before age 25 years. A kidney biopsy was performed in 15 patients. In all cases, light microscopy showed sclerosis of renal glomeruli, manifesting as FSGS or diffuse mesangial sclerosis (Table 1, Supplemental Table 1, Figure 1, and Supplemental Figure 5). Electron microscopy furthermore revealed partial podocyte foot process effacement, one of the hallmark symptoms of nephrotic syndrome (Figure 1 and Supplemental Figure 5). We termed these previously unrecognized monogenic causes of SRNS as NPHS17 (NUP85), NPHS18 (NUP133), and NPHS19 (NUP160).

In a consanguineous Pakistani family with 3 affected children with congenital microcephaly, we discovered a homozygous nonsense mutation (c.916C $>\mathrm{T}$, p.Arg306*) in the gene NUP37 (NM_024057.3) (Table 1 and Supplemental Figure 6). NUP37 encodes an essential component of the Y complex of the NPC. Segregation analysis was performed when possible, and was compatible with a recessive mode of inheritance (Supplemental Figure 6). All 3 patients showed congenital microcephaly ( -5 to -8 standard deviations [SD]), mild intellectual disability, cerebellar vermis hypoplasia, and clinodactyly of the fifth finger (Table 1). Proteinuria was not present in these patients, and renal ultrasound examination did not reveal any abnormalities (Table 1).

Mutations from SRNS patients impair intermolecular interactions between NPC components. NUP107, NUP85, NUP133, and NUP160 are components of the $\mathrm{Y}$ complex, whose molecular structure has recently been modeled into the outer ring scaffold of the NPC (11) (Figure 2A). To investigate the pathogenicity of alleles that we discovered in SRNS patients, we gleaned from available structural data (Protein Data Bank, pdb: 5A9Q) (11) that 1 mutation of NUP133, p.Ser974Arg, and 1 mutation of NUP107, p.Tyr889Cys, were located in the interacting $\alpha$-helix between the 2 proteins (Figure 2A). NUP85 mutations were positioned within a region for which no molecular structure of the human $\mathrm{Y}$ complex is available yet (Figure 2A).

We used cDNA constructs reflecting the mutations identified in patients with SRNS (Table 1) and performed half-endogenous coimmunoprecipitation (coIP) experiments in HEK293T cells. These experiments demonstrated that, as predicted based on the structural data, the alleles p.Tyr889Cys of NUP107 and p.Ser974Arg of NUP133 weakened the interaction between NUP107 and its direct binding partner NUP133 (Figure 2, B and C). Other missense alleles of NUP107 or NUP133 did not 
A

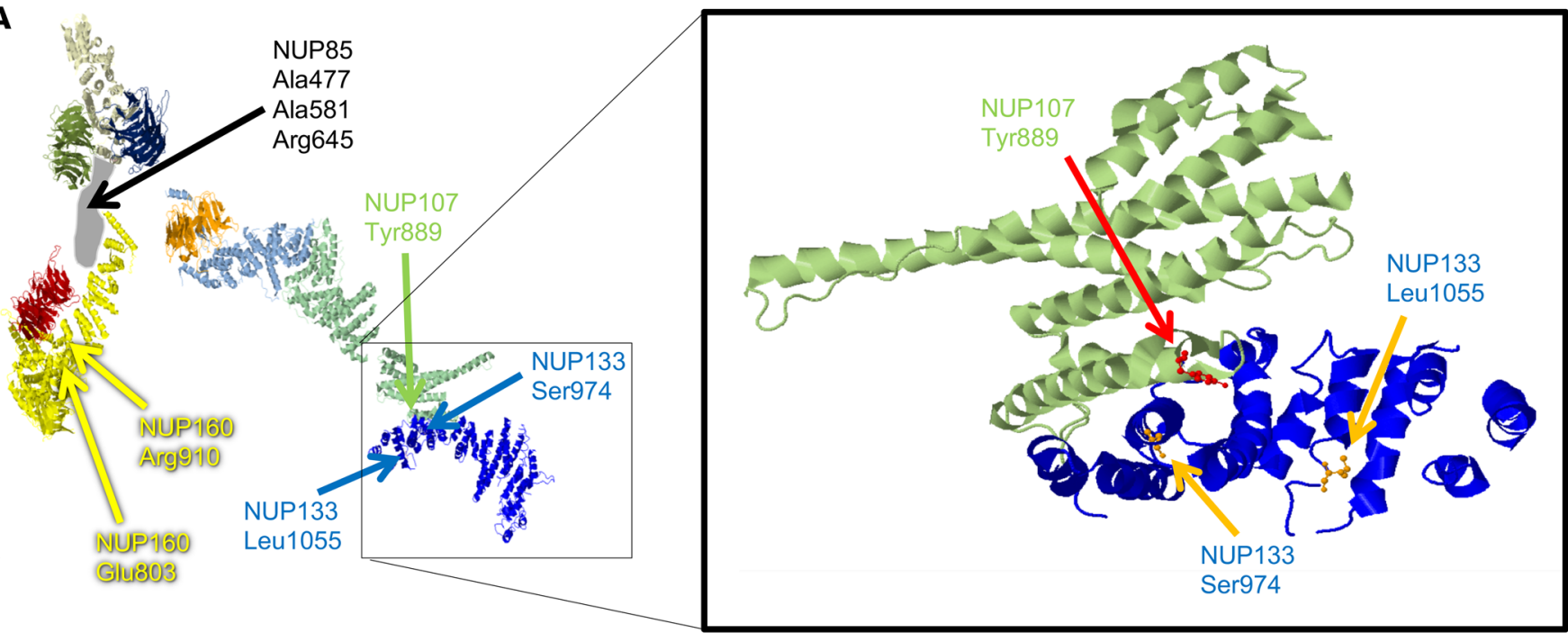

B
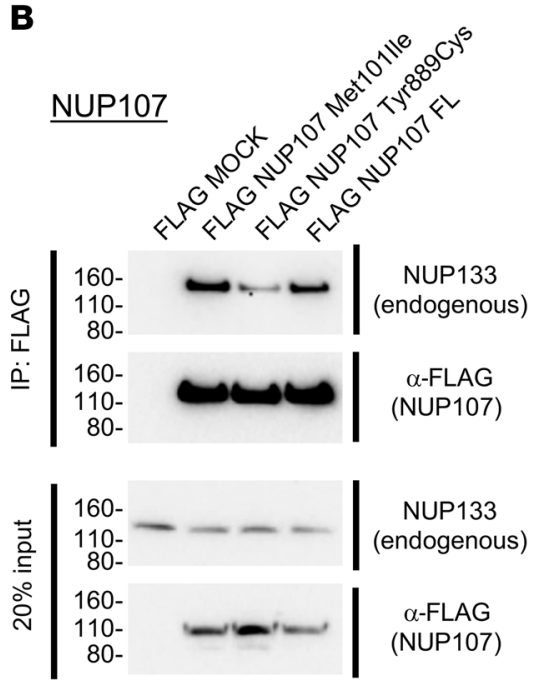

D

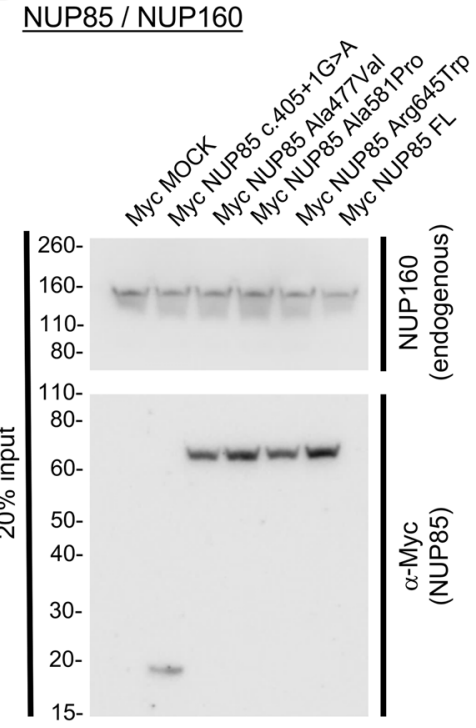

C

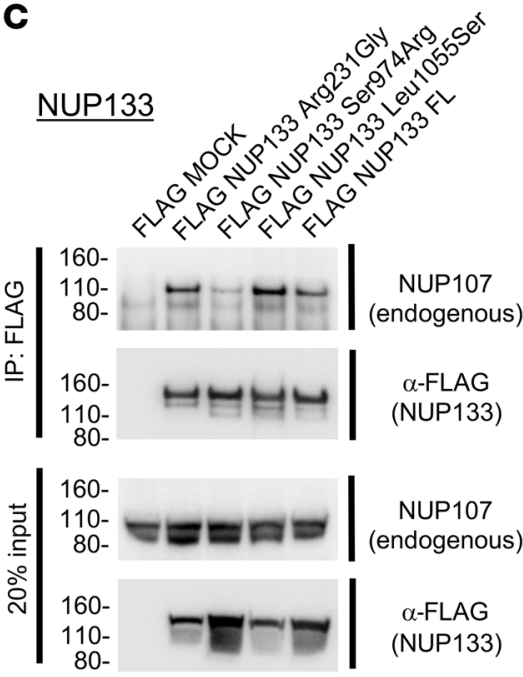

Figure 2. Mutations of NUP107, NUP85, and NUP133 weaken protein-protein interactions within the NPC. (A) The published structure of the Y complex of the NPC (11) was used to determine the localization of amino acid residues that we found altered in individuals with SRNS. Left: The 3D structure of the $Y$ complex (pdb: 5A9Q): NUP133 (dark blue), NUP107 (light green), NUP96 (light blue), SEC13 (orange), SEH1L (violet), NUP85 (light gray), NUP43 (dark green), NUP160 (yellow), and NUP37 (red). Inset on right: The 3D structure of the interface region between NUP107 and NUP133 (pdb: 3CQC). The C-terminal part of NUP85 could not be fully resolved experimentally; a gray area indicates its predicted position. Residues Ala477, Ala581, and Arg645 of NUP85 (gray) and Glu803 of NUP160 (yellow) are located within incompletely resolved areas, and their positions are estimated. Note that amino acid residues Tyr889 of NUP107 (light green) and Ser974 of NUP133 (dark blue) point toward the interaction interface. (B) N-terminally FLAG-tagged NUP107 wild-type (WT) or mutant cDNA was overexpressed in HEK293T cells. Coimmunoprecipitation (colP) demonstrates that the missense mutation Tyr889Cys weakens the interaction with endogenous NUP133. (C) A colP experiment using $\mathrm{N}$-terminally FLAG-tagged WT or mutant NUP133 cDNA demonstrates that the missense mutation Ser974Arg weakens the interaction with endogenous NUP107. As expected based on structural data, the 2 other missense mutations (Arg231Cly and Leu1055Ser) do not alter the NUP107-NUP133 interaction. (D) N-terminally Myc-tagged WT or mutant NUP85 CDNA was overexpressed in HEK293T cells. ColP using an antibody against endogenous NUP160 shows that the missense mutations Ala581Pro and Arg645Trp of NUP85 weaken the interaction between the 2 proteins. $\mathrm{FL}$, full-length; MOCK, empty vector. ColP experiments in $\mathbf{B}-\mathbf{D}$ were confirmed in 3 independent experiments. 
interfere with the interaction between the 2 proteins. We furthermore show that the missense mutations p.Ala581Pro and p.Arg645Trp of NUP85 weakened the interaction with its binding partner NUP160 (Figure 2D). The obligatory splice site mutation c. $405+1 G>A$ of NUP85 is predicted to cause skipping of exon 5, thus resulting in a frameshift with premature truncating of the encoded protein. We modeled this mutation in a cDNA construct and found that it completely abrogated the interaction between NUP85 and NUP160 (Figure 2D). Confirmatory experiments for all coIPs using differently tagged fusion proteins are shown in Supplemental Figure 7. Next, we aimed to investigate the impact of an isolated depletion of SRNS-relevant NUPs on other components of the NPC. To address this question, we generated immortalized human podocyte cell lines with doxycycline-inducible CRISPR/Cas9 mediated knockout of NUP107, NUP85, or NUP133 (Supplemental Figures 8-10 demonstrate successful depletion of targeted proteins). We used an inducible system because sustained knockout of any of the 3 investigated NUP genes had strong effects on cell viability, which we feared would mask any specific effects resulting from the knockout. Using the inducible CRISPR/Cas9 system, we could instead initiate depletion of the specific gene of interest shortly before the experiment and thereby study its direct effect. In this setting, we did not observe significant cell death. Interestingly, we found that short-term depletion of NUP85 or NUP133 had very little effect on the protein levels of other components of the NPC (Supplemental Figure 11).

Morpholino knockdown of Xenopus nup107, nup85, or nup133 causes defects in glomerulogenesis. At the 2-cell stage, Xenopus embryos were injected with a morpholino oligonucleotide targeting nup85, nup107, or nup133. In Xenopus, cell divisions are holoblastic (complete); therefore, we can do lateralized injections of 1 cell at the 2-cell stage such that only half of the animal is affected during development. In this setting, the uninjected side can act as an internal control. In contrast, the initial cleavages of zebrafish embryos are mesoblastic (incomplete); as a consequence, injected mRNAs cannot be localized to just 1 cell. For this reason, Xenopus is an ideal system in which to test rescue especially of lateralized structures such as the kidney (Figure $3 \mathrm{~A}$ ). At stage $35-37$, we detected the pronephros using atp1a1 as a marker in whole-mount in situ hybridization experiments. At this stage, the proximal portion of the pronephros develops a convoluted shape that subsequently straightens out as it extends to the posterior portion of the embryo.

We categorized defects in pronephros morphology based on their severity (Figure 3B). Compared with controls (Figure 3, C and D), morpholino knockdown resulted in abnormal pronephros morphology in $65 \%$ of nup 85 morphants (Figure 3, E vs. F, and K), $38 \%$ of nup107 morphants (Figure 3, G vs. H, and K), and $86 \%$ of nup133 morphants (Figure 3, I vs. J, and K). Morpholino knockdown of pax8, which encodes an essential transcription factor in renal development, was used as a positive control to validate this approach (Supplemental Figure 12A). We used the gene NUP155 as a negative control, because the phenotype caused by NUP155 mutations, monogenic atrial fibrillation (33), does not involve the kidney. As expected, knockdown of nup155 did not result in a renal phenotype in the Xenopus model (Supplemental Figure 12B). These findings suggest that the nucleoporins Nup85, Nup107, and Nup133 are required for renal development in Xenopus.
In order to test the specificity of the knockdown experiment and to assess the pathogenicity of human mutations, we used rescue experiments (Supplemental Figure 13). We first injected nup85, nup107, or nup133 morpholinos at the 1-cell stage to deplete these proteins throughout the embryo. At the 2-cell stage, we then injected 1 cell of these morphants with WT or mutant human mRNA of NUP85, NUP107, or NUP133, respectively (Supplemental Figure 13A). While WT mRNA rescued the abnormal kidney morphology seen in morphant kidneys, mutant mRNA reflecting the human SRNS mutations resulted in an impaired restoration of renal morphology (Supplemental Figure 13, B-D). Only the p.Ala477Val variant of NUP85 did not show a significantly reduced rescue efficiency. Based on this result, we would predict that this allele may have some residual function, and that it may represent a relatively mild allele as compared with the others. Interestingly, the phenotype in those patients was not notably different.

CRISPR/Cas9 knockout of NUP107, NUP85, or NUP133 increases the level of active $C d c 42$. Dysregulation of the Rho-like small GTPases RhoA, Rac1, and Cdc42, resulting in impaired actin dynamics, plays an important role in the pathogenesis of monogenic SRNS (34-39). Interestingly, we observed that CRISPR/Cas9-mediated knockout of NUP107, NUP85, or NUP133 increased the formation of filopodia in immortalized human podocytes (Figure 4, A and B), suggesting that loss of function of these genes alters the podocyte's cytoskeleton. Because Cdc42 is known to induce filopodia formation, if active (40), we performed the colorimetric Cdc42 G-LISA Activation Assay to determine the cellular level of active Cdc42. We found that in comparison with control cells (Cas9 expression but no guide RNA [gRNA]), CRISPR/Cas9 knockout of any of the 3 genes significantly increased Cdc42 activity (Figure 4C). However, when using the IncuCyte system to assess the migration rate of podocytes, we did not find a significant difference between control and knockout cells (Supplemental Figure 14).

Truncating mutations of nup107 or nup 85 in zebrafish induce early lethality, while an in-frame mutation of nup107 does not affect survival. We designed gRNAs targeting exon 2 of nup107, the zebrafish ortholog (NM_001030167.1). Using CRISPR/Cas9 technology, we then generated 2 different stable zebrafish lines, one with a proteintruncating "null" allele (c.50_56del7, p.Thr81Argfs*74) and one with an in-frame mutation, most likely acting as a hypomorphic allele (c.137_139del3, p.Ala46delAla). After het $\times$ het in-crossing, we monitored survival twice daily and generated Kaplan-Meier survival curves. Zebrafish with a homozygous truncating mutation of nup107 showed early lethality at 5 days postfertilization (dpf) (Figure 5A) and demonstrated severe developmental malformations, including small eyes, ventral body axis curvature, periorbital edema, and total body edema (Figure 5, C- G, and Table 2). These phenotypes developed at $4 \mathrm{dpf}$ before death on $5 \mathrm{dpf}$. Zebrafish were born at Mendelian ratios. None of the 29 larvae homozygous for the null allele p.Thr81Argfs ${ }^{*} 74$ were phenotypically normal. Conversely, none of the 42 heterozygous larvae and none of the 34 WT larvae developed any overt phenotype (Table 2). In contrast to the truncating allele, the hypomorphic mutation of nup107 did not affect survival of zebrafish larvae (Figure 5B) and did not result in any overt phenotype (data not shown).

We then used CRISPR/Cas9 technology and a gRNA targeting exon 4 of nup85 (NM 001003625.1) to generate a 
A

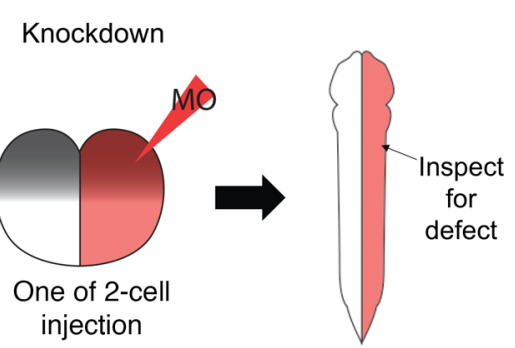

B
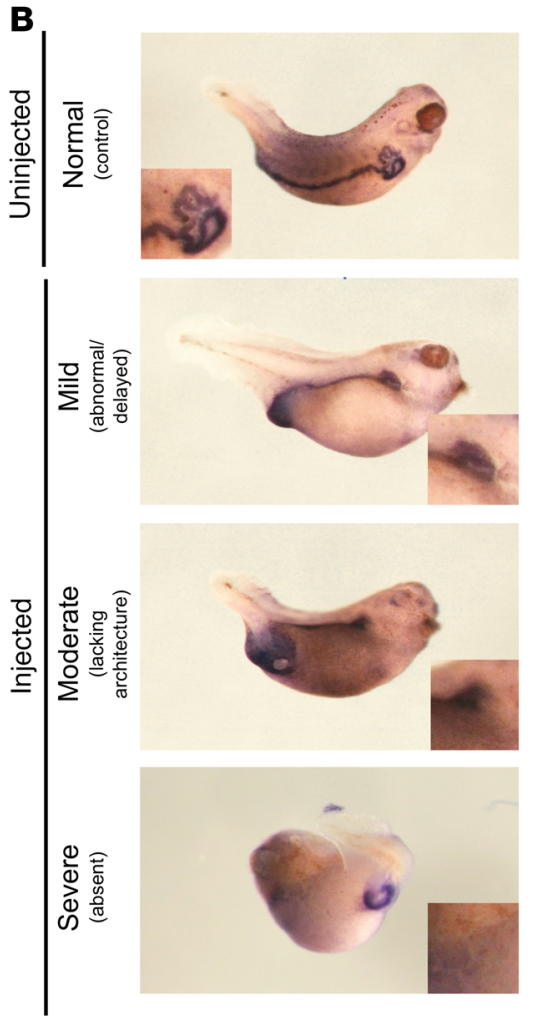

$\mathbf{K}$

Severity of pronephros abnormalities

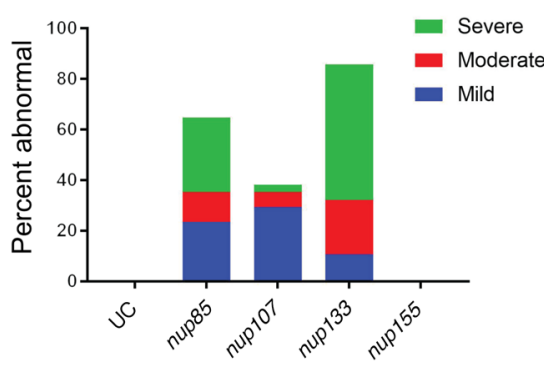

Control
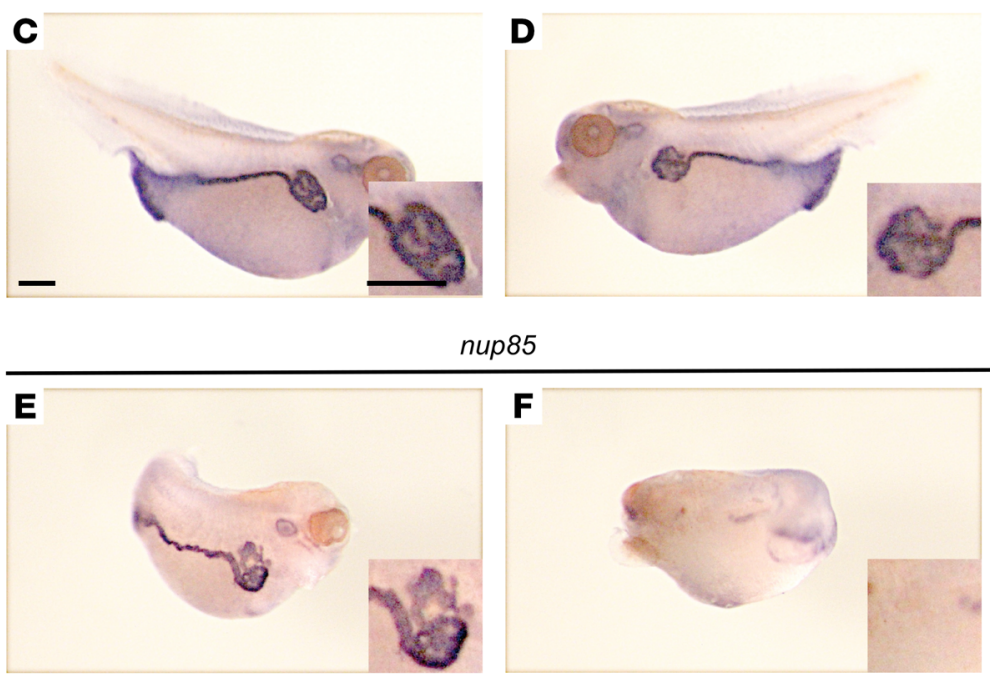

nup 107
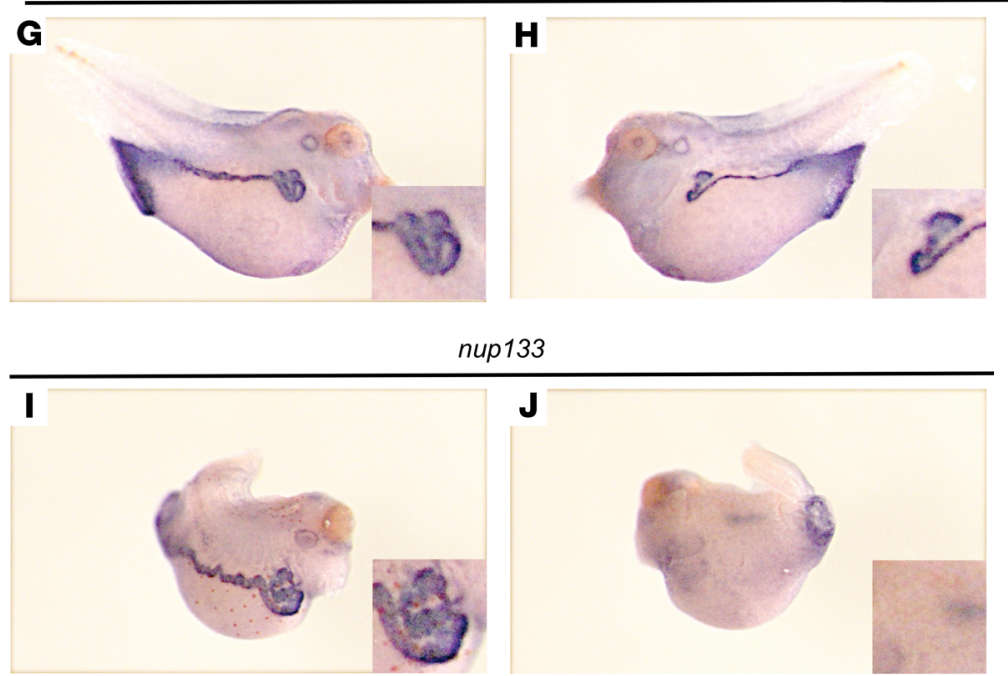

Figure 3. Morpholino knockdown of nup85, nup107, or nup133 in Xenopus embryos causes defects in glomerulogenesis. Xenopus embryos were injected with morpholino oligonucleotides (MOs) targeting nup85, nup107, or nup133 at the 2-cell stage. Abnormalities in pronephric development, specifically improper formation of the convoluted pronephric duct, were scored at stages 35-37. The pronephros was detected using whole-mount in situ hybridization and atp1a1 as a marker. (A) Schematic of the experimental setup, in which injection of $\mathrm{MO}$ into 1 cell of a 2-cell embryo allows for 1 side of the embryo to develop normally, while the other half serves as an internal control for developmental phenotypes. (B) Based on the severity of the phenotype, morphants were sorted into 4 groups: normal, mild phenotype (delayed or decreased convolution of the pronephric duct, top panel), moderate phenotype (loss of the characteristic pronephric architecture, middle panel), and severe phenotype (pronephros entirely absent, bottom panel). Shown here are examples for each category. (C-J) Left panels display the uninjected control side. Right panels display the injected side. Scale bars: $200 \mu \mathrm{m}$. (C and D) Control embryo (injected with nontargeting MO) displaying appropriate pronephric morphology for this stage (see B). (C) Uninjected. (D) Injected. (E and F) MO knockdown of nup85 results in abnormalities of pronephric development. (E) Uninjected. (F) Injected. (G and H) MO knockdown of nup107 causes developmental defects of the pronephros. Note the developmental delay and simplification of the convoluted pronephric duct. (G) Uninjected. (H) Injected. (I and J) Morpholino knockdown of nup133 causes pronephric developmental abnormalities. (I) Uninjected. (J) Injected. (K) Phenotypes in nup85, nup107, nup133, or nup155 morphants were categorized into the 4 groups. Note that as compared with uninjected controls (UC, $n=32$ ), nup85 (65\% abnormal, $n=34$ ) and nup133 ( $86 \%$ abnormal, $n=28$ ) morphants more frequently display severe phenotypes, while nup107 (38\% abnormal, $n=34$ ) morphants tend to show milder phenotypes. All experiments were performed at least twice. 
A
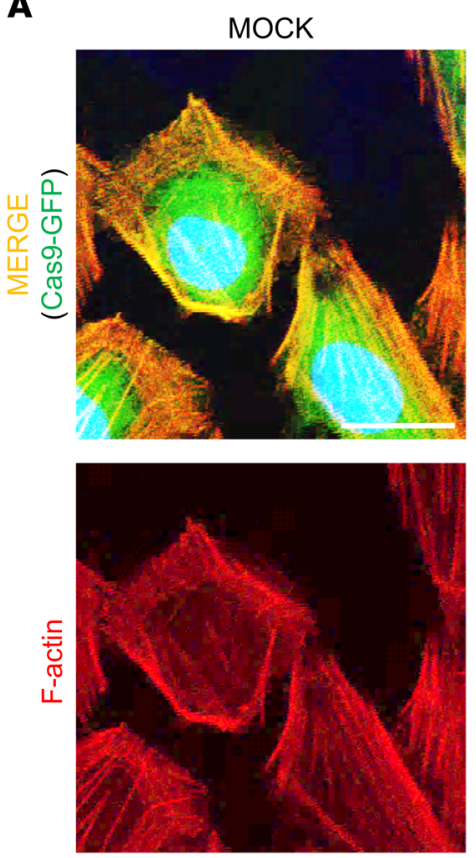

B

Percentage of podocytes with filopodia $(n=356)$

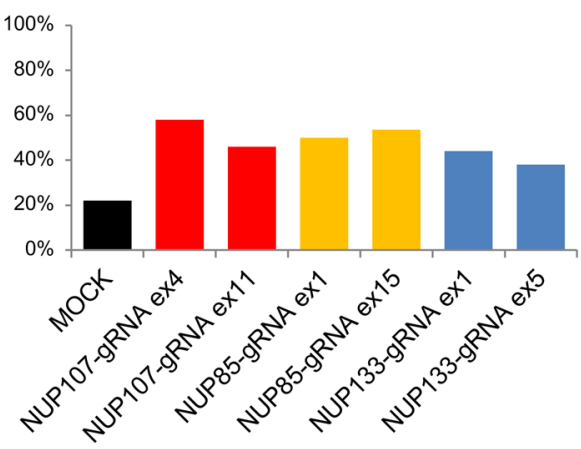

NUP107-gRNA ex4
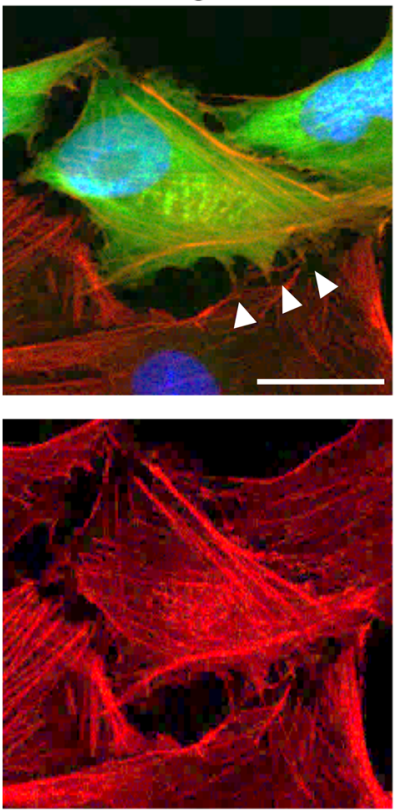

NUP85-gRNA ex15
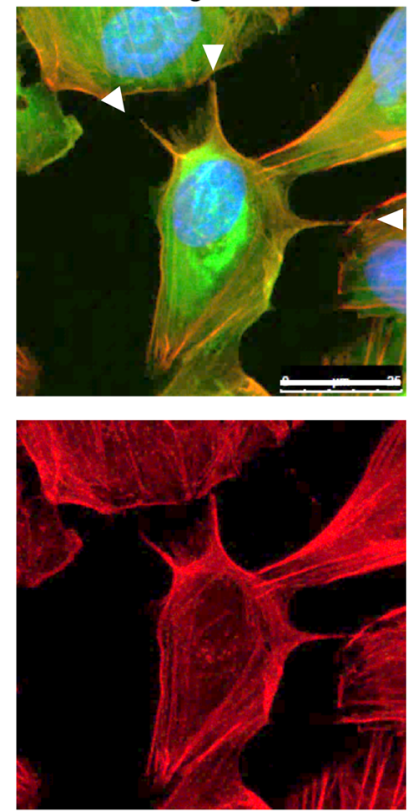

NUP133-gRNA ex5
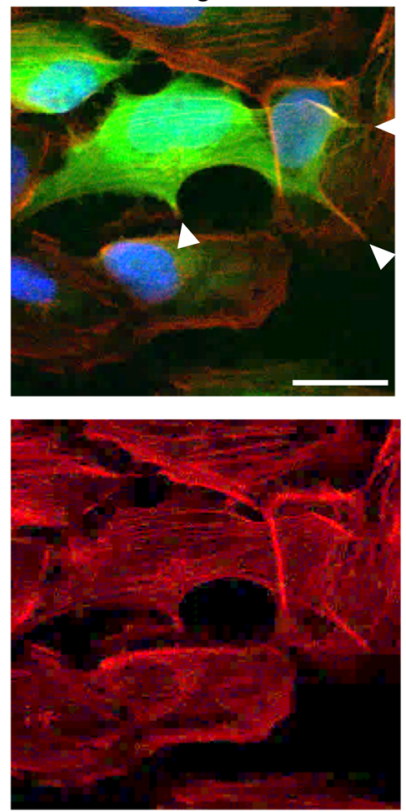

c

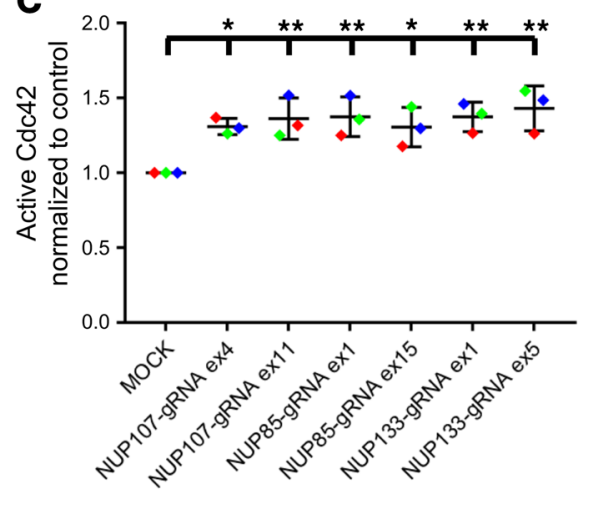

Figure 4. CRISPR/Cas9-mediated knockout of NUP107, NUP85, or NUP133 induces filopodia formation and increases active Cdc42 in human podocytes. Immortalized human podocytes underwent lentiviral transduction with a plasmid expressing a Cas9-GFP fusion construct under the control of a doxycyclineinducible promoter and a single gRNA. For each gene, 2 different cell lines were generated expressing gRNAs against NUP107 (targeting exon 4 or 11), NUP85 (targeting exon 1 or 15), or NUP133 (targeting exon 1 or 5), respectively. Experiments were performed 72 hours after induction of Cas9 expression with doxycycline $(1 \mu \mathrm{g} / \mathrm{ml}$ ). (A) We stained immortalized human podocytes expressing empty vector (MOCK) or individual gRNAs targeting NUP107, NUP85, or NUP133 with phalloidin to detect F-actin fibers. Podocytes that had either 3 actin-based protrusions or 1 filamentous protrusion of more than one-quarter of the cell body were quantified as "filopodia positive." Representative images showing "filopodia-negative" control cells and NUP107-, NUP85-, or NUP133knockout podocytes that exhibited filopodia (arrowheads). Scale bars: $25 \mu \mathrm{m}$. The result was confirmed in 3 independent experiments. (B) Quantification of approximately 50 cells for each condition resulted in 22\% of MOCK-expressing cells with filopodia (11/50), in contrast to 58\% (29/50) for NUP107 gRNA exon 4 (ex4) and 46\% (23/50) for NUP107 gRNA ex11; 50\% (25/50) for NUP85 gRNA ex1 and 54\% (30/56) for NUP85 gRNA ex15; and 44\% (22/50) for NUP133 gRNA ex1 and 38\% (19/50) for NUP133 gRNA ex5. Note that knockout podocytes show increased filopodia formation. (C) Using the colorimetric G-Lisa Cdc42 Activation Assay Biochem Kit (Cytoskeleton), we demonstrate an increase in the active state of Cdc42 following CRISPR/Cas9-mediated knockout of NUP107, NUP85, or NUP133 in human podocytes. Data points represent 3 independent experiments (highlighted in different colors) and are displayed with mean and SD. $P$ values calculated by 1 -way ANOVA are indicated in the figure as ${ }^{*} P<0.05$; ${ }^{*} P<0.01$.

zebrafish line carrying the truncating mutation p.Arg107Cysfs ${ }^{\star} 15$ of nup85, the zebrafish ortholog of human NUP85. As observed with nup107 knockout, homozygous nup85-knockout fish showed early lethality and died at day $8 \mathrm{dpf}$ (Supplemental Figure 15A). The phenotypic features of nup85-knockout fish resembled those of nup107-knockout fish, including small eyes, body axis curvature, and edema (Supplemental Figure 15, B-E, and Supplemental Table 2).
To test for renal anomalies and for a kidney-specific phenotype, we used transmission electron microscopy (TEM) to investigate the ultrastructure of the renal glomerulus and used H\&E staining to study renal histology. For the 2 null alleles of nup107 and nup85, we investigated 2 homozygous versus 2 heterozygous fish by TEM and 8 homozygous versus 8 heterozygous fish by renal histology for each gene. We detected significant tissue decay within the glomerular region, but also globally throughout the 

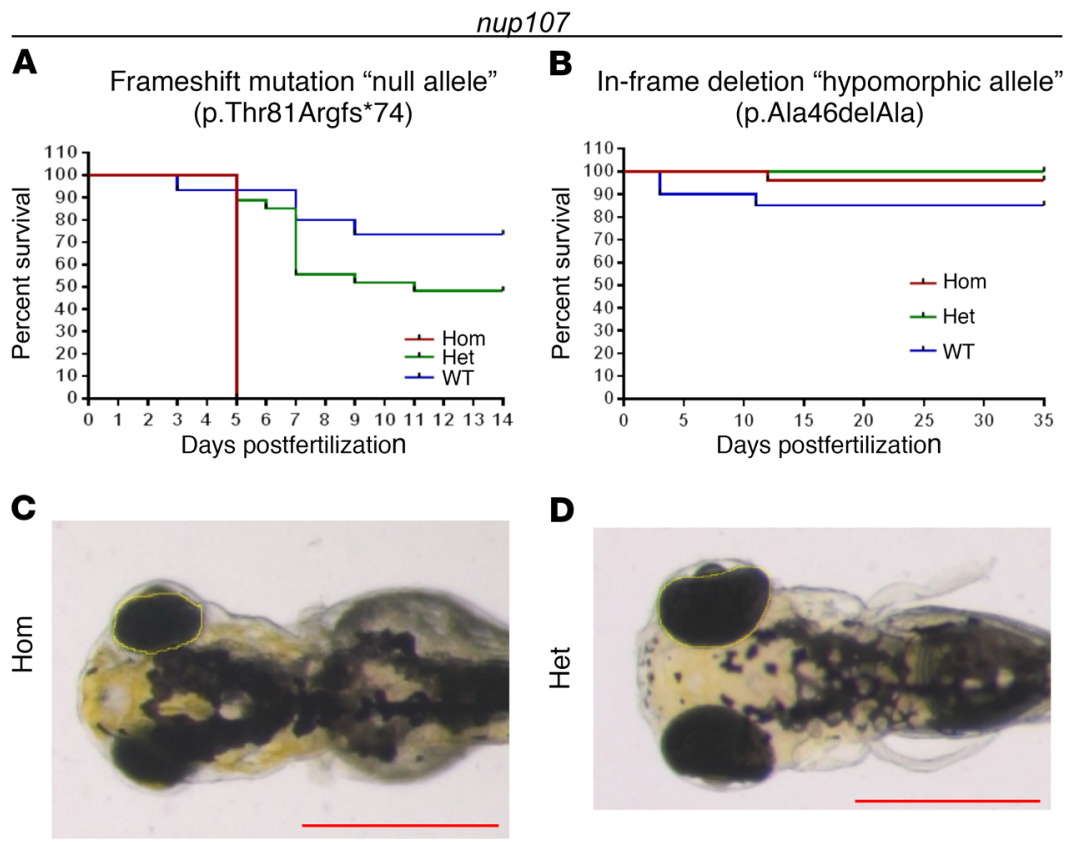

D
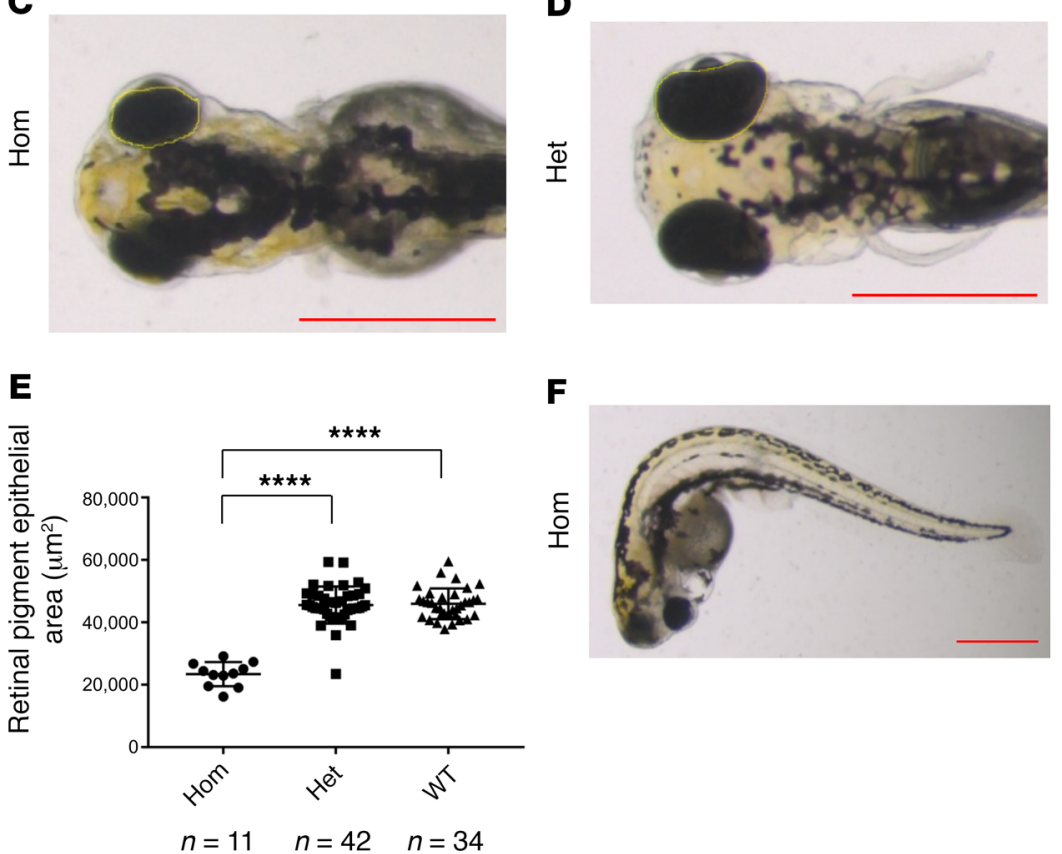

G

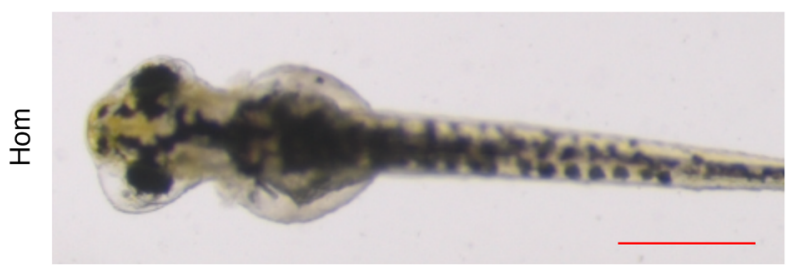

Figure 5. A truncating mutation but not a hypomorphic mutation of nup107 causes early lethality and developmental defects in zebrafish. Zebrafish lines with mutations of nup107 were generated using CRISPR/Cas9 technology. Lethality following het $x$ het in-crossing was monitored twice daily over the indicated periods. Genotyping was performed in all fish and was compatible with Mendelian ratios. (A) Kaplan-Meier survival curves of 86 larvae demonstrate that homozygous (hom) larvae carrying the frameshift mutation p.Thr81Argfs* 74 of nup107 died before $5 \mathrm{dpf}$, contrary to heterozygous (het) and wild-type (WT) controls ( $n=26$ hom, 39 het, 21 WT). (B) Kaplan-Meier survival curves of a zebrafish line carrying a hypomorphic mutation of nup107 (p.Ala46delAla). Note that, contrary to the truncating allele, this in-frame deletion of nup107 does not impair survival of homozygous larvae compared with WT fish or heterozygous clutch mates ( $n=14$ hom, 27 het, 15 WT). (C-C) Phenotypes of homozygous nup107-knockout larvae (p.Thr81Argfs*74) on day $4 \mathrm{dpf}$. Specifically, the phenotype included small eyes, ventral body axis curvature, and peripheral as well as periorbital edema. (C and $\mathbf{D})$ Yellow circumferences drawn around the pigmented area of the eyes of knockout fish (C) versus heterozygous clutch mates (D) assess eye size using Imagej. (E) Quantification of eye size measurements (see $\mathbf{C}$ and $\mathbf{D}$ ) demonstrates significantly smaller eyes in homozygous fish compared with heterozygous or WT clutch mates. One-way ANOVA with a standard confidence interval of $95 \%$ results in $F(2,84)=84.72 ; P<0.0001$. Two-tailed $P$ values (Šidák's multiple-comparisons test) are shown in the figure $\left.{ }^{* * * *} P<0.001\right)$. (F) Representative image showing ventral body axis curvature in a homozygous knockout fish. (G) Representative image displaying body and periorbital edema in a homozygous knockout fish. For quantification of $\mathbf{F}$ and G, see Table 2. Scale bars in C, D, F, and G: $500 \mu \mathrm{m}$. larva. We are therefore hesitant to interpret these findings as a specific podocyte phenotype, but rather assume that the loss of an essential protein induces injury in all cell types.

Primary patient cell lines carrying protein-truncating mutations of NUP37 and NUP107 display changes in NPC composition and nuclear morphology. We obtained primary cell lines from 2 patients with microcephaly and with mutations of NUP37 and NUP107, respectively. In dermal fibroblasts from a patient carrying the p.Arg306* allele of NUP37, we demonstrated that at the mRNA (Supplemental Figure 6E) and protein level (Figure 6, A and D) NUP37 was reduced in comparison with control cells. Reduced NUP37 protein levels resulted in codepletion of other components of the Y complex, such as NUP107 and NUP160 (Figure 6, A-D). A similar effect was observed in a lymphoblastoid cell line
(LCL) from a patient with the p.Met101Ile mutation of NUP107 (Supplemental Figure 16, A-C). These findings suggest that, as previously described $(28,29)$, proteins of the Y complex depend on each other for protein stability. Interestingly, in both cell lines residual amounts of the mutant protein were present.

When investigating the consequences of reduced NUP37 amounts for NPC density, we found a reduced intensity of the mAb414 antibody signal, suggesting a lower number of nuclear pores in mutant fibroblasts as compared with control cells (Figure 6, $\mathrm{E}$ and $\mathrm{F}$ ). Using antibodies detecting heterochromatin (anti-HP1 $\beta$; Figure 6G) and nucleoli (anti-fibrillarin; Figure 6, H and I), we demonstrated an alteration of chromatin organization and nucleolar morphology in NUP37 mutant fibroblasts. TEM confirmed this observation (Figure 6, J-M, arrowheads). Additionally, we found 
the perinuclear space to be widened and irregular (Figure 6, L and $\mathrm{M}$, arrows), and we detected bulbous invasions of the nuclear envelope (Figure 6M, star) in NUP37 mutant fibroblasts. Notably, the cell proliferation rate of NUP37-mutant as compared with control fibroblasts was reduced (Supplemental Figure 6F). We admit that, as mutations in NUP genes predominantly manifest in postmitotic, epithelial tissues, proliferating fibroblasts have important limitations as a model system.

\section{Discussion}

In summary, we here discovered recessive mutations in the genes NUP85, NUP133, and NUP160 as 3 monogenic causes of SRNS. Interestingly, all 3 genes and the gene NUP107, in which we found 3 pathogenic alleles, encode proteins that are components of a distinct subunit of the nuclear pore complex (NPC), the so-called Y complex or outer ring subunit of the NPC. In coIP experiments, we demonstrated that 5 human mutations altered protein-protein interaction between direct binding partners in the NPC. Using morpholino knockdown in Xenopus larvae, we provided evidence that these NUPs play a distinct role in renal development. The renal phenotype of Xenopus morphants could be rescued by WT, but not mutant, mRNA, thus demonstrating pathogenicity for the alleles that we identified in patients with SRNS. In contrast to all other tested alleles, the p.Ala477Val allele of NUP85 rescued the phenotype, thereby suggesting that in this case the function of the encoded protein was at least partially preserved. This result may be explained by (a) the mild chemical difference between WT and altered amino acid (alanine vs. valine) and (b) the fact that this residue was not conserved between the human and the frog protein. As there was no difference in the severity of the phenotype in affected patients, we assume that the function of the human protein may still be significantly altered by this mutation. In cell culture studies, we observed that knockout of NUP107, NUP85, or NUP133 in immortalized human podocytes changed the activity state of the Rho-like small GTPase Cdc42, a pathway that is known to be relevant to monogenic forms of SRNS $(34-37,41)$. When generating stable, transgenic zebrafish lines using CRISPR/ Cas9 technology, we observed that null alleles of nup107 or nup85 caused severe developmental malformations and early lethality. In contrast, an in-frame mutation of nup107 was compatible with survival and did not cause apparent developmental anomalies, thus reflecting the allelism seen in patients with NUP107 mutations.

Accumulating evidence suggests that apart from their universal function in nucleocytoplasmic transport, certain NUP proteins may have additional, individual functions in cell differentiation and during development $(16,42)$. By performing morpholino knockdown experiments, we generated the first evidence to our knowledge that Nup107, Nup85, and Nup133 are required for renal development in Xenopus. The observation that knockdown of nup155 did not result in abnormal pronephros morphology suggests that this function is specific to certain NUPs. With one exception, mutant mRNAs, reflecting alleles that we found mutated in SRNS patients, were less efficient than WT mRNA in restoring the pronephros morphology in Xenopus morphants. Based on these observations, we hypothesize that SRNS development in patients with mutations of NUP107, NUP85, or NUP133 partially reflects an alteration of specific functions of those NUPs during renal development.
The Rho-like small GTPases RhoA, Rac1, and Cdc42 are regulators of the actin cytoskeleton (43). Podocytes depend on a highly dynamic and tightly regulated actin cytoskeleton to generate and maintain their actin-based foot processes and the slit membrane of the renal glomerular filter (39). Dysregulation of these Rho-like small GTPases has been observed in several monogenic forms (34-37, 41) and mouse models of SRNS $(38,44)$. We therefore propose that the observed increase in the level of active Cdc42 upon knockout of NUP85, NUP107, or NUP133 may contribute to the pathogenesis of SRNS in patients with mutations in these genes. Mechanistically, further studies will be needed to elucidate the molecular link between NPC disruption and dysregulation of Cdc 42 activity. However, based on currently available data, it seems likely that alterations in NUPs are linked to the activity state of $\mathrm{Cdc} 42$ by an indirect effect. This functional link may be mediated, e.g., by nuclear import of specific regulatory elements or by nuclear export of distinct RNA species.

Prominent phenotypic features of nup107- and nup85-knockout zebrafish larvae included edema, ventral body axis curvature, and diminished eye size. This phenotype recapitulated a previously published transcription activator-like effector nuclease-mediated (TALEN-mediated) nup107-knockout zebrafish line (18). Before death, knockout zebrafish showed profound decay in all tissues, including the glomerular area. However, because of its global character, we are hesitant to interpret these findings as a tissue-specific phenotype. We assume that knockout larvae die prematurely as a result of the complete lack of Nup85 and Nup107, two essential proteins, before a specific renal phenotype may develop.

Summarizing all findings, we suggest the following explanation for the observed genotype-phenotype correlation in patients with NUP107 mutations: While missense mutations likely have little impact on protein stability and cellular protein level, the p.Met101Ile allele of NUP1O7 resulted in a reduced amount of NUP107 protein with consecutive changes in NPC composition and nuclear organization. In conclusion, we propose that 3 types of mutations in essential NUP-encoding genes exist: (a) completeloss-of-function mutations that cause embryonic lethality and are therefore not observed in human patients; (b) partial-loss-offunction mutations that reduce the amount of the encoded proteins and cause syndromic, developmental phenotypes, but are compatible with survival; and (c) strictly hypomorphic missense mutations that impair only specific aspects of the protein's functionality without affecting its overall function and therefore result in a distinct, organ-restricted phenotype.

Mutations in the genes NUP93, NUP205, and NUP107, encoding proteins of the NPC, were recently described as causing SRNS (21-24). It was vexing that mutations in these essential genes of high evolutionary conservation and universal relevance gave rise to such a distinct phenotype. By identifying 3 additional genes that encode scaffold proteins of the NPC and cause SRNS, if mutated, we provide further evidence that distinct, hypomorphic mutations in this group of fundamental genes may specifically impact podocytes and may manifest in a cell type-specific manner.

\section{Methods}

Study participants. We collected blood samples and pedigree information after informed consent from individuals with SRNS or their guardians. 

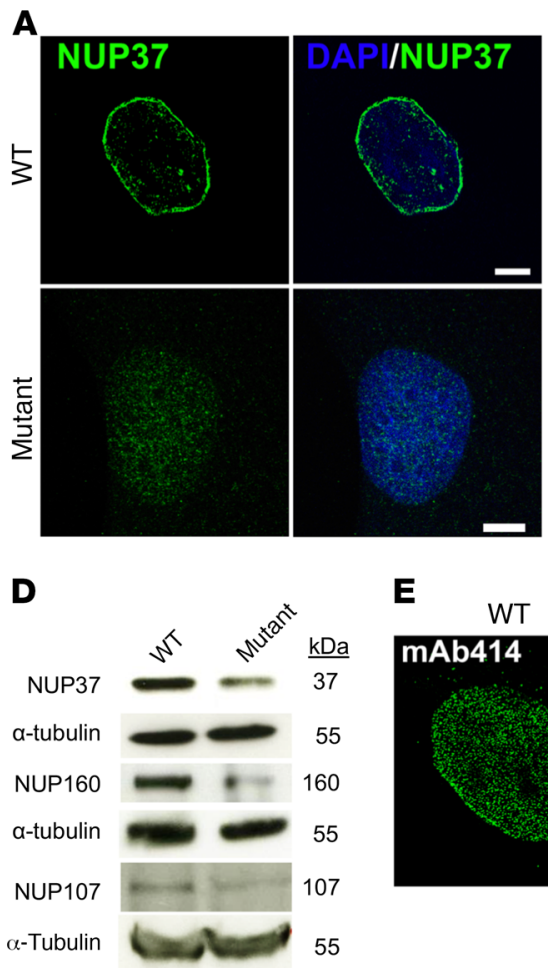

B

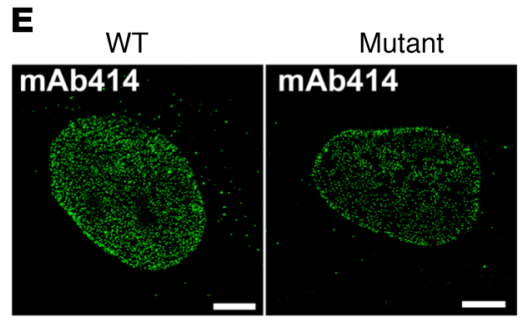

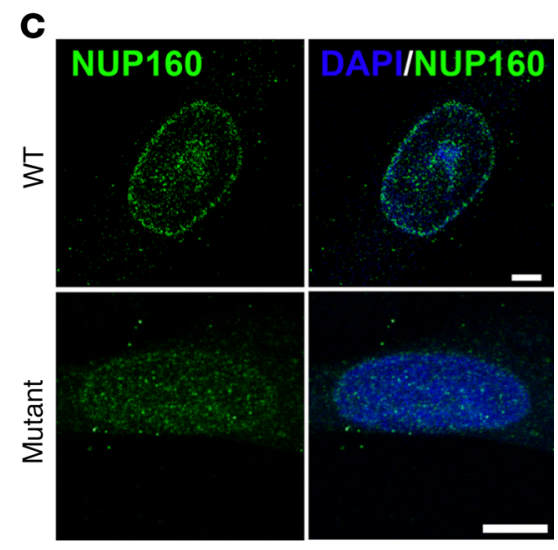

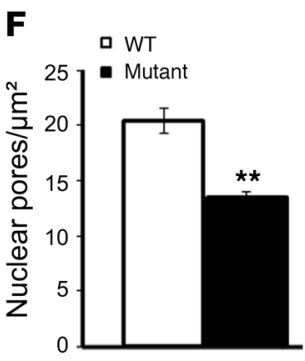

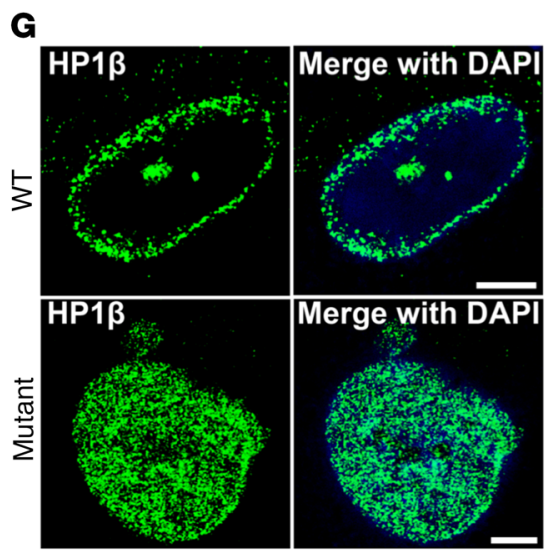

J

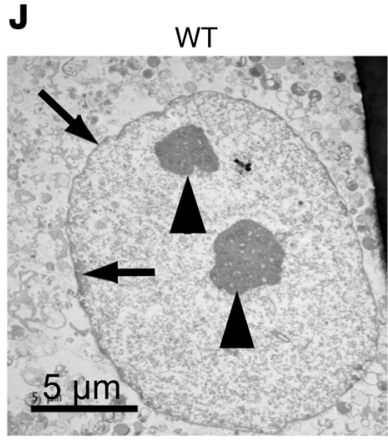

K

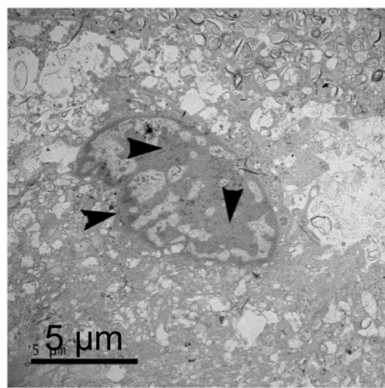

$H$

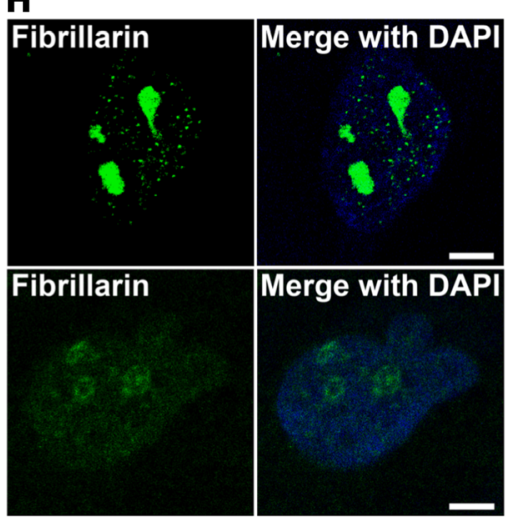

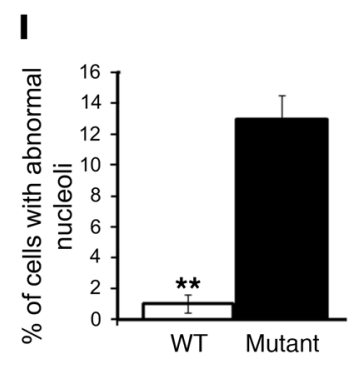

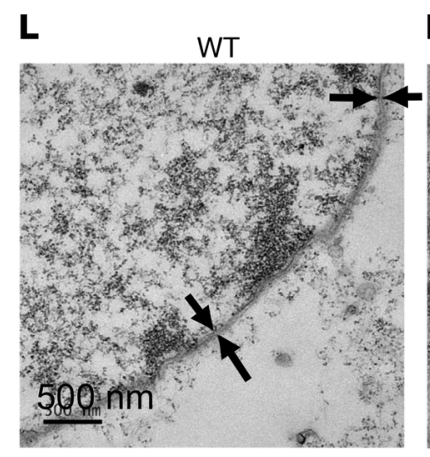

M

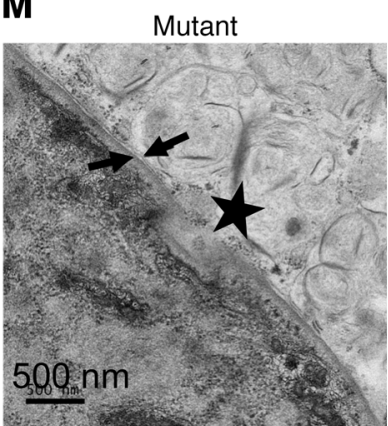


Figure 6. Impact of a NUP37 mutation on the composition of the NPC and on nuclear structure. A primary fibroblast cell line from patient PN-2 with the homozygous truncating mutation p.Arg306* of NUP37 (mutant) was compared with control fibroblasts (WT). DAPI stains DNA (blue). (A-C) Confocal microscopy of immunostaining for NUP37, NUP107, and NUP160 in mutant versus control fibroblasts. (D) Immunoblotting of NUP37, NUP160, and NUP107 in mutant versus control fibroblasts. $\alpha$-Tubulin serves as a loading control. Note that protein levels are reduced in mutant fibroblasts. (E) Immunostaining with an antibody against several FG-repeat nucleoporins (mAb414) in control (left) versus mutant (right) fibroblasts. (F) Quantification of $\mathbf{E}$ demonstrates a significant reduction in the number of NPCs per square micrometer in mutant versus control cells. Data were obtained for 100 cells from 3 different experiments. Error bars denote SEM. $P=0.0048$ (Student's $t$ test). (G) Immunostaining of HP1 $\beta$ (green), labeling heterochromatin, demonstrates an altered pattern in mutant versus control fibroblasts. (H) Fibrillarin (green) was used to stain nucleoli. Note that fibrillarin staining was more dispersed in mutant fibroblasts. (I) Quantification of 150 cells from 3 independent experiments demonstrates a significantly increased percentage of nuclei with abnormal nucleoli in mutant versus control cells. Error bars represent SEM. $P=0.0018$ (Student's $t$ test). Scale bars in A-C, E, G, and $\mathbf{H}: 5 \mu \mathrm{m}$. (J-M) TEM images of control versus mutant fibroblasts. In control (WT) cells, a regular nuclear envelope (arrow outside the nucleus in J) and well-arranged heterochromatin in the proximity of the nuclear envelope (arrow inside the nucleus in J) can be seen. Note that the nuclear architecture of mutant fibroblasts is altered; specifically, (a) there is abnormal arrangement of heterochromatin and nucleoli (arrowheads, J vs. $\mathbf{K})$; (b) the perinuclear space is widened and irregular (arrows, L vs. M); and (c) bulbous invasions of the nuclear envelope were observed (star, $\mathbf{M}$ ). Scale bars are defined in each image. Immunofluorescence experiments (A-C, E, $\mathbf{G}$, and $\mathbf{H}$ ) and immunoblotting results $(\mathbf{D})$ were confirmed in 3 independent experiments. ${ }^{* *} P<0.01$.

Homozygosity mapping. Homozygosity mapping was performed using the GeneChip Human Mapping 250k StyI Array, Affymetrix, and the programs GENEHUNTER $2.1(45,46)$ and ALLEGRO $(47)$ as described previously (48). Alternatively, homozygosity mapping was generated based on whole exome sequencing data using the Genome Analysis Tool Kit (49) and the program Homozygosity Mapper (50). For families PN-1 and PN-2, the Illumina HumanCoreExome 24 v1.1 array (Illumina) was used for genotyping. Further analysis was performed as described previously (51). See Supplemental Methods for a more detailed description.

Whole exome sequencing. Whole exome sequencing was performed on genomic DNA isolated from blood lymphocytes or saliva and subjected to exome capture using Agilent SureSelect human exome capture arrays (Agilent Technologies) followed by next-generation sequencing on a HiSeq Illumina sequencing platform. Sequence reads were mapped to the human reference genome (NCBI build 37/hg19) using CLC Genomics Workbench (version 6.5.2; CLC bio). Variants with minor allele frequencies greater than $1 \%$ in the dbSNP database (version 142) were excluded. Remaining variants were evaluated and ranked based on established criteria $(3,4,52)$ and pedigree information. For families $\mathrm{PN}-1$ and $\mathrm{PN}-2$, whole exome sequencing was performed using the Agilent version 6 enrichment kit and the Illumina HiSeq 4000 sequencing system (paired-end reads, $2 \times 75 \mathrm{bp}$ ) as described previously (53). For filtration and prioritization of variants, we used the Cologne Center for Genomics' VARBANK database and analysis tool kit (54).

High-throughput mutation analysis by multiplex PCR with subsequent next-generation sequencing. As previously described $(30,31)$, we used 48.48 Access Array microfluidic technology (Fluidigm) and $2 \times 250 \mathrm{bp}$ paired-end sequencing on an Illumina MiSeq instrument (Illumina) to sequence the coding regions of 19 genes that encode different NUPs in 2,164 individuals with nephrotic syndrome. Bioinformatic analysis was conducted using CLC Genomics Workbench software (version 6.5.2; CLC bio). Identified mutations were confirmed by Sanger sequencing. Segregation analysis was performed whenever possible (Table 1).

In silico modeling of NUP85, NUP107, and NUP133 mutations. The model of the human Y complex (outer ring subunit of the NPC) was based on published data available from the Protein Data Bank (pdb). Figure 2A is based on pdb structures 5A9Q (11) and 3CQC (55). We used the open-source program Jmol (an open-source Java viewer for chemical structures in 3D; http://www.jmol.org/) to generate the 3D projections that are shown in Figure 2.

cDNA cloning and stable CRISPR/Cas9 cell lines. The following human cDNA constructs were used for this study: NUP107 (NM_020401.3),NUP85(NM_024844.4), and NUP133(NM_018230.2). The following expression vectors were used for cell culture experiments: pRK5-N-Myc, pDEST69-N-Flag, and pCDNA6.2-N-GFP. The inducible CRISPR/Cas9 backbone (TLCV2) was a gift from Adam Karpf (Eppley Institute, University of Nebraska Medical Center, Omaha, Nebraska, USA) (Addgene plasmid 87360). This backbone is based on LentiCRISPR-v2 and expresses Cas9-P2A-GFP under the control of a doxycycline-inducible promoter (tight TRE promoter). Additionally, a single gRNA and a puromycin-resistance cassette are expressed constitutively. Stable cell lines were generated using lentiviral transduction and puromycin selection $(4 \mu \mathrm{g} / \mathrm{ml})$ for more than 7 days. Cas 9 expression was induced 72 hours before all experiments using doxycycline (1 $\mu \mathrm{g} / \mathrm{ml})$. Western blotting and immunofluorescence experiments confirmed a reduction in the expression level of targeted proteins (Supplemental Figures 8-10).

gRNA sequences are provided in Supplemental Table 3.

Antibodies are listed in Supplemental Table 4.

Cell lines. Immortalized human podocytes were a gift from Moin Saleem (University of Bristol, Bristol, United Kingdom) and have been extensively characterized in previous publications (56). HEK293T cells were purchased from the ATCC biological resource center and were used for virus production and coIP experiments. Cells were tested monthly for mycoplasma contamination. Primary cell lines, i.e., human dermal fibroblasts or lymphoblastoid cell lines (LCLs) from patients with a homozygous mutation of NUP107 (PN-1) or NUP37 (PN-2), were generated as described previously $(57,58)$.

Coimmunoprecipitation. CoIP experiments were performed in HEK293T cells. Thirty-six hours after cDNA transfection, protein lysates were harvested, and 450-600 $\mu \mathrm{g}$ of protein was incubated for 4 hours with (a) EZview Red Anti-c-Myc Affinity Gel (Sigma-Aldrich), (b) EZview Red ANTI-FLAG M2 Affinity Gel (Sigma-Aldrich), or (c) Chromotek-GFP-Trap Agarose Beads (Allele Biotechnology) depending on the expressed fusion protein and as indicated in the figures. Before elution, beads were washed 5 times with IP lysis buffer (Thermo Fisher Scientific). The result of each coIP study was confirmed in 3 independent experiments and was repeated using differently tagged fusion proteins.

Phenotyping in Xenopus embryos. Antisense morpholino oligonucleotides (MOs) or mRNAs were injected either at the 1-cell stage or into 1 cell of the 2-cell embryo as previously described (59). Sequences of MOs are provided in Supplemental Table 3. We generated mRNA of WT and mutated human sequences using a T7 promoter template in the pcDNA6.2-N-GFP backbone. In vitro capped mRNA was gener- 
Table 2. Quantification of phenotypes in homozygous nup107knockout zebrafish larvae (p.Thr81Argfs*74)

$\begin{array}{lccccc} & \begin{array}{c}\text { Small } \\ \text { eyes }\end{array} & \begin{array}{c}\text { Ventral } \\ \text { curvature }^{\mathrm{A}}\end{array} & \begin{array}{c}\text { Body } \\ \text { edema }^{\mathrm{B}}\end{array} & \begin{array}{c}\text { Periorbital } \\ \text { edema }^{\mathrm{B}, \mathrm{C}}\end{array} & \text { Normal } \\ \text { Homozygous } & 11 & 18 & 7 & 5 & 0 \\ \text { Heterozygous } & 0 & 0 & 0 & 0 & 42 \\ \text { WT } & 0 & 0 & 0 & 0 & 34\end{array}$

Definitions of assessed phenotypes: Small eyes: assessed by measurement of the surface area of the retinal pigmented epithelium in dorsal view images (see yellow drawn circumferences in Figure 5, C and D). Body edema: defined as a clear, fluid-filled space around the torso in dorsal view images (see Figure 5G). Periorbital edema: defined as a clear, fluid-filled space before and/or behind the retinal pigment epithelium of the eyes in the dorsal view (see Figure 5G). ${ }^{\mathrm{A}}$ Eye size and body and periorbital edema could not by assessed in ventrally curved fish (see Figure $5 F ; n=18$ ). ${ }^{B}$ All fish with either body edema or periorbital edema had small eyes. 'Five of seven homozygous p.Thr81Argfs* 74 fish with small eyes and body edema also had periorbital edema.

ated using the T7 mMessage machine kit (Ambion, Thermo Fisher Scientific). Five nanograms of MOs were injected for knockdown experiments, 10 ng of MOs for rescue experiments, and 200 pg of mRNA. To label the pronephros, we detected Xenopus atp1a1 expression by generating a digoxigenin-labeled antisense probe using the T7 High Yield RNA Synthesis kit (New England Biolabs, E2040S). Embryos were collected at stage 35-37. Whole mount in situ hybridization was done as previously described (59). We qualitatively assessed pronephric morphology based on atp1a1 expression. Abnormalities were designated as mild, moderate, or severe (Figure 3B). All experiments were performed a minimum of 2 times, and numbers stated in graphs are the composite of multiple experiments. Statistical significance of glomerular abnormalities and rescues was evaluated by Fisher's exact tests using GraphPad Prism version 7.00 (GraphPad Software). In all figures, statistical significance was defined as $P<0.05$. $P$ values, as indicates by asterisks, are defined in the respective figure legends. See Supplemental Methods for a more detailed description.

$G$-Lisa for active Cdc42. Active Cdc42 was assessed in immortalized human podocytes stably expressing doxycycline-inducible Cas9P2A-GFP and single gRNAs targeting NUP107, NUP85, or NUP133 or no gRNA (MOCK). The measurements were performed using the colorimetric G-LISA Cdc42 Activation Assay Kit (Cytoskeleton Inc.) following the manufacturer's instructions. Results are given as absorbance value at $490 \mathrm{~nm}$ and are normalized to the control condition (MOCK). Individual data points in Figure 4 represent the mean of 2 technical replicates derived from 3 independent experiments and are displayed in different colors with mean and SD. Statistical significance was calculated using 1-way ANOVA with a standard confidence interval of $95 \%$. F values resulting from ANOVA and 2-tailed $P$ values derived from Šidák multivariate analysis are indicated. Statistical analysis was performed using GraphPad Prism. $P<0.05$ was considered statistically significant.

Immunofluorescence, confocal microscopy, and quantification of filopodia. Confocal imaging in human podocytes was performed using the Leica SP5X system with an upright DM6000 microscope, and images were processed with the Leica AF software suite. Experiments for filopodia quantification were performed in human podocytes sta- bly expressing the inducible Cas9-P2A-GFP system with empty vector (MOCK) or single gRNA targeting NUP107, NUP85, or NUP133. Fifty cells for each condition were analyzed. The GFP signal confirmed expression of Cas9-P2A-GFP. Phalloidin stained F-actin fibers and was used to identify filopodia. To score cells as "filopodia positive" or "filopodia negative," we used the 2 following criteria: (a) cells that displayed at least 1 filamentous protrusion with a length of more than one-quarter of the cell diameter, or (b) cells that had 3 or more actinbased, spike-like protrusions. Cells that fulfilled one or both criteria were scored as filopodia-positive cells.

Images in Figure 6 and Supplemental Figure 16A were taken with a confocal laser scanning microscope (TCS SP8 gSTED, Leica Microsystems) or a confocal microscope (LSM TCS SP5, Leica Microsystems). Nuclear pore density was analyzed using the "particle analysis" tool of ImageJ (version 1.51z; NIH). Samples were prepared for transmission electron microscope as reported previously (60) and viewed with a transmission electron microscope (JEOL JEM2100PLUS). Quantitative PCR experiments were performed as described previously (53). The cell proliferation assay was performed as described previously (57).

Assessment of the cell migration rate of immortalized human podocytes with CRISPR/Cas9-mediated knockout of NUP85, NUP107, or NUP133. We used the IncuCyte video microscopy system (Essen Biosciences). Podocytes were seeded on a 96-well plate and grown to confluence, and a standardized scratch wound was made using the Woundmaker device according to protocol. Wound closure was recorded by live cell imaging every hour for 20-24 hours. Data analysis was performed using the IncuCyte 96-well Kinetic Cell Migration and Invasion Assay software module. Individual data points are presented as mean \pm SD resulting from at least triplicate measurements. Experiments were repeated 3 times independently.

Generation and characterization of stable zebrafish lines with CRISPR/ Cas9-mediated introduction of hypomorphic or truncating mutations of nup107 or nup85. We designed single gRNA targeting exon 2 of nup107 and exon 4 of nup85 using the CHOPCHOP online tool (https:// chopchop.rc.fas.harvard.edu) (61). Single gRNA and recombinant Cas9 protein were injected at the 1-cell stage. Successful mutagenesis was determined by a T7 endonuclease assay as described previously (62). Positive clutches were raised to adulthood and outcrossed against WT fish. Founder lines were genotyped using Sanger sequencing. We chose 1 line with a truncating mutation of nup107 (c.50_56del7, p.Thr81Argfs ${ }^{*} 74$ ), 1 line with an in-frame deletion mutation of nup107 (c.137_139del3, pAla46delAla), and 1 line carrying a null allele of nup85 (c.323_332delGAGCCTGTATinsGCT, p.Arg107Cysfs¹5).

Phenotyping in zebrafish larvae. We generated Kaplan-Meier survival curves by comparing WT, heterozygous, and knockout larvae. During the experimental period, we monitored the phenotype twice daily. All dead larvae and all surviving fish were genotyped at the end of the experiment using Sanger sequencing.

Phenotyping was performed at $4 \mathrm{dpf}$ for nup107 fish and $7 \mathrm{dpf}$ for nup85 fish. Zebrafish with ventral body axis curvature were imaged from a lateral view. Therefore, other parameters were not assessed in those fish. Larvae with normal body axis were imaged from a dorsal view. To determine eye size, we measured the surface of the black pigmented epithelium in the retina using the program ImageJ. We defined body edema as the presence of a clear, fluid-filled space around the torso in dorsal view, and periorbital edema as the presence 
of a clear, fluid-filled space before and/or behind the retinal pigment epithelium of the eyes. Statistical analysis of eye size was performed using GraphPad Prism to calculate 1-way ANOVA test with multiple comparisons and a standard confidence interval of $95 \%$. The resulting 2-tailed $P$ values and $F$ values are indicated. Zebrafish experiments were performed in Danio rerio, strain l-fabp: VDBP-GFP (AB). This line was a gift from Weibin Zhou (University of Michigan, Ann Arbor, Michigan, USA). For additional details see Supplemental Methods.

Study approval. Approval for human subjects research was obtained from Institutional Review Boards of the University of Michigan (Ann Arbor, Michigan, USA), Boston Children's Hospital (Boston, Massachusetts, USA), and the National Institute for Biotechnology and Genetic Engineering, Faisalabad, Pakistan. All national and institutional guidelines for the care and use of laboratory animals were followed. Zebrafish experiments were approved by the Boston Children's Hospital Institutional Animal Care and Use Committee (IACUC; protocol 15-12-3087R). Xenopus experiments were approved by the Yale IACUC (protocol 2015-11035).

\section{Author contributions}

SL, DS, AD, JR, WT, SA, HYG, SS, JAL, JKW, TH, EW, AJM, HH, AM, BB, S Motameny, JA, S Mane, MSH, PN, RPL, and FH performed whole exome evaluation and mutation analysis. DAB performed coimmunoprecipitation experiments and in vitro experiments in podocytes. WA confirmed the coimmunoprecipitation experiments. RS, A Kolb, TJS, JMS, CAH, TMK, and KE performed zebrafish experiments. JM, CPL, and MKK performed Xenopus experiments. MA, $\mathrm{KS}$, AIAK, AAN, and MSH performed in vitro experiments in primary patient cell lines. SL, DS, AD, DAB, JR, WT, HMF, DPG, LK, SH, KAA, MH, NM, RE, ES, FSA, QS, HX, A Khan, SSW, GA, MU, SMB, SG, CA, and $\mathrm{FH}$ recruited patients and gathered detailed clinical information for the study. All authors critically reviewed the paper. FH conceived of and directed the project. DAB and FH wrote the paper.

\section{Acknowledgments}

We are grateful to study participants and their families for their contribution. We thank the Yale Center for Mendelian Genomics for whole exome sequencing analysis (U54HG006504). For technical help, we thank Ramona Casper and Maria Stumpf. We also thank the CECAD Imaging Facility, University of Cologne, for the electron and confocal laser microscopy data. We furthermore thank the Regional Computing Center of the University of Cologne (RRZK) for providing computing time on the DFG-funded High Performance Computing (HPC) system CHEOPS as well as support.

This research was supported by grants from the NIH to $\mathrm{FH}$ (DK076683) and to MKK and CPL (HL124402). JM was supported by the Yale Medical Scientist Training Program (MSTP) (NIH grant T32 GM07205) and NIH grant T32 GM007223 from the Yale Predoctoral Program in Cellular and Molecular Biology. PN and AAN acknowledge support from the Center for Molecular Medicine Cologne. MSH was supported by the Köln Fortune Program of the Faculty of Medicine, University of Cologne. TJS was supported by grant Jo 1324/1-1 from the Deutsche Forschungsgemeinschaft (DFG). EW was supported by the German National Academy of Sciences Leopoldina (LPDS-2015-07). AJM was supported by the Harvard Stem Cell Institute, Kidney Group. WT was supported by the ASN (American Society of Nephrology) Foundation for Kidney Research. TH was supported by the German Research Foundation, DFG fellowship (HE 7456/1-1). CA was supported by grants from the European Union's Seventh Framework Programme (FP7/2007-2013/no 305608, EURenOmics), the Fondation Recherche Medicale (DEQ20150331682), and the Investments for the Future program (ANR-10-IAHU-01). SG was supported by the MD-PhD program of Imagine Institute (ANR10-IAHU-01 and Fondation Bettencourt-Schueller). TMK was supported by a Post-Doctoral Fellowship award from the KRESCENT Program, a national kidney research training partnership of the Kidney Foundation of Canada, the Canadian Society of Nephrology, and the Canadian Institutes of Health Research. MA, AM, and SSW were supported by the Higher Education Commission of Pakistan. AIAK is supported by King Saud University.

Address correspondence to: Friedhelm Hildebrandt, Boston Children's Hospital, Enders 561, Harvard Medical School, 300 Longwood Avenue, Boston, Massachusetts 02115, USA. Phone: 617.355.6129; Email: friedhelm.hildebrandt@childrens. harvard.edu.
1. Trautmann A, et al. Long-term outcome of steroid-resistant nephrotic syndrome in children. J Am Soc Nephrol. 2017;28(10):3055-3065.

2. Smith JM, Stablein DM, Munoz R, Hebert D, McDonald RA. Contributions of the Transplant Registry: The 2006 Annual Report of the North American Pediatric Renal Trials and Collaborative Studies (NAPRTCS). Pediatr Transplant. 2007;11(4):366-373.

3. Lovric S, Ashraf S, Tan W, Hildebrandt F. Genetic testing in steroid-resistant nephrotic syndrome: when and how? Nephrol Dial Transplant. 2016;31(11):1802-1813.

4. Vivante A, Hildebrandt F. Exploring the genetic basis of early-onset chronic kidney disease. Nat Rev Nephrol. 2016;12(3):133-146.

5. Machuca E, Benoit G, Antignac C. Genetics of nephrotic syndrome: connecting molecular genetics to podocyte physiology. Hum Mol Genet. 2009;18(R2):R185-R194.
6. Wiggins RC. The spectrum of podocytopathies: a unifying view of glomerular diseases. Kidney Int. 2007;71(12):1205-1214.

7. Sadowski CE, et al. A single-gene cause in $29.5 \%$ of cases of steroid-resistant nephrotic syndrome. JAm Soc Nephrol. 2015;26(6):1279-1289.

8. Bierzynska A, et al. Genomic and clinical profiling of a national nephrotic syndrome cohort advocates a precision medicine approach to disease management. Kidney Int. 2017;91(4):937-947.

9. Giglio S, et al. Heterogeneous genetic alterations in sporadic nephrotic syndrome associate with resistance to immunosuppression. JAm Soc Nephrol. 2015;26(1):230-236.

10. Beck M, Hurt E. The nuclear pore complex: understanding its function through structural insight. Nat Rev Mol Cell Biol. 2017;18(2):73-89.

11. von Appen A, et al. In situ structural analysis of the human nuclear pore complex. Nature. 2015;526(7571):140-143.
12. Kosinski J, et al. Molecular architecture of the inner ring scaffold of the human nuclear pore complex. Science. 2016;352(6283):363-365.

13. Wozniak R, Burke B, Doye V. Nuclear transport and the mitotic apparatus: an evolving relationship. Cell Mol Life Sci. 2010;67(13):2215-2230.

14. Chatel G, Fahrenkrog B. Nucleoporins: leaving the nuclear pore complex for a successful mitosis. Cell Signal. 2011;23(10):1555-1562.

15. Del Viso F, et al. Congenital heart disease genetics uncovers context-dependent organization and function of nucleoporins at cilia. Dev Cell. 2016;38(5):478-492.

16. Lupu F, Alves A, Anderson K, Doye V, Lacy E. Nuclear pore composition regulates neural stem/ progenitor cell differentiation in the mouse embryo. Dev Cell. 2008;14(6):831-842.

17. Jacinto FV, Benner C, Hetzer MW. The nucleoporin Nup153 regulates embryonic stem cell pluripotency through gene silencing. Genes Dev. 
2015;29(12):1224-1238.

18. Zheng X, et al. Loss of zygotic NUP107 protein causes missing of pharyngeal skeleton and other tissue defects with impaired nuclear pore function in zebrafish embryos. J Biol Chem. 2012;287(45):38254-38264.

19. Shang $X$, et al. Molecular cloning of the rice field eel Nup93 with predominant expression in gonad and kidney. Yi Chuan Xue Bao. 2006;33(1):41-48.

20. Reza N, Khokha MK, Del Viso F. Nucleoporin gene expression in Xenopus tropicalis embryonic development. Int J Dev Biol. 2016;60(4-6):181-188.

21. Braun DA, et al. Mutations in nuclear pore genes NUP93, NUP205 and XPO5 cause steroidresistant nephrotic syndrome. Nat Genet. 2016;48(4):457-465.

22. Miyake N, et al. Biallelic mutations in nuclear pore complex subunit NUP107 cause earlychildhood-onset steroid-resistant nephrotic syndrome. Am J Hum Genet. 2015;97(4):555-566.

23. Rosti RO, et al. Homozygous mutation in NUP107 leads to microcephaly with steroid-resistant nephrotic condition similar to Galloway-Mowat syndrome. JMed Genet. 2017;54(6):399-403.

24. Park E, Ahn YH, Kang HG, Miyake N, Tsukaguchi $\mathrm{H}$, Cheong HI. NUP107 mutations in children with steroid-resistant nephrotic syndrome. Nephrol Dial Transplant. 2017;32(6):1013-1017.

25. Zhou W, et al. FAN1 mutations cause karyomegalic interstitial nephritis, linking chronic kidney failure to defective DNA damage repair. Nat Genet. 2012;44(8):910-915.

26. Hildebrandt F, et al. A systematic approach to mapping recessive disease genes in individuals from outbred populations. PLoS Genet. 2009;5(1):e1000353.

27. Alazami AM, et al. Accelerating novel candidate gene discovery in neurogenetic disorders via whole-exome sequencing of prescreened multiplex consanguineous families. Cell Rep. 2015;10(2):148-161.

28. Boehmer T, Enninga J, Dales S, Blobel G, Zhong H. Depletion of a single nucleoporin, Nup107, prevents the assembly of a subset of nucleoporins into the nuclear pore complex. Proc Natl Acad Sci U S A. 2003;100(3):981-985.

29. Walther TC, et al. The conserved Nup107-160 complex is critical for nuclear pore complex assembly. Cell. 2003;113(2):195-206.

30. Halbritter J, et al. High-throughput mutation analysis in patients with a nephronophthisisassociated ciliopathy applying multiplexed barcoded array-based PCR amplification and next-generation sequencing. JMed Genet. 2012;49(12):756-767.

31. Halbritter J, et al. Identification of 99 novel mutations in a worldwide cohort of 1,056 patients with a nephronophthisis-related ciliopathy. Hum
Genet. 2013;132(8):865-884.

32. Vivante A, et al. Exome sequencing discerns syndromes in patients from consanguineous families with congenital anomalies of the kidneys and urinary tract. J Am Soc Nephrol. 2017;28(1):69-75.

33. Zhang $\mathrm{X}$, et al. Mutation in nuclear pore component NUP155 leads to atrial fibrillation and early sudden cardiac death. Cell. 2008;135(6):1017-1027.

34. Gee HY, et al. ARHGDIA mutations cause nephrotic syndrome via defective RHO GTPase signaling. J Clin Invest. 2013;123(8):3243-3253.

35. Gee HY, et al. KANK deficiency leads to podocyte dysfunction and nephrotic syndrome. JClin Invest. 2015;125(6):2375-2384.

36. Gee HY, et al. FAT1 mutations cause a glomerulotubular nephropathy. Nat Commun 2016;7:10822

37. Boyer O, et al. INF2 mutations in Charcot-MarieTooth disease with glomerulopathy. N Engl J Med 2011;365(25):2377-2388.

38. Scott RP, et al. Podocyte-specific loss of Cdc42 leads to congenital nephropathy. JAm Soc Nephrol. 2012;23(7):1149-1154.

39. Faul C, Asanuma K, Yanagida-Asanuma E, Kim K, Mundel P. Actin up: regulation of podocyte structure and function by components of the actin cytoskeleton. Trends Cell Biol. 2007;17(9):428-437.

40. Nobes CD, Hall A. Rho, rac and cdc42 GTPases: regulators of actin structures, cell adhesion and motility. Biochem Soc Trans. 1995;23(3):456-459.

41. Gee HY, et al. Mutations in EMP2 cause childhoodonset nephrotic syndrome. Am J Hum Genet. 2014;94(6):884-890.

42. D’Angelo MA, Gomez-Cavazos JS, Mei A, Lackner DH, Hetzer MW. A change in nuclear pore complex composition regulates cell differentiation. Dev Cell. 2012;22(2):446-458.

43. Hall A. Rho GTPases and the control of cell behaviour. Biochem Soc Trans. 2005;33(pt 5):891-895.

44. Blattner SM, et al. Divergent functions of the Rho GTPases Rac1 and Cdc42 in podocyte injury. Kidney Int. 2013;84(5):920-930.

45. Kruglyak L, Daly MJ, Reeve-Daly MP, Lander ES. Parametric and nonparametric linkage analysis: a unified multipoint approach. Am J Hum Genet. 1996;58(6):1347-1363.

46. Strauch K, Fimmers R, Kurz T, Deichmann KA, Wienker TF, Baur MP. Parametric and nonparametric multipoint linkage analysis with imprinting and two-locus-trait models: application to mite sensitization. Am J Hum Genet. 2000;66(6):1945-1957.

47. Gudbjartsson DF, Jonasson K, Frigge ML, Kong A. Allegro, a new computer program for multipoint linkage analysis. Nat Genet. 2000;25(1):12-13.
48. Chaki M, et al. Exome capture reveals ZNF423 and CEP164 mutations, linking renal ciliopathies to DNA damage response signaling. Cell. 2012;150(3):533-548.

49. Van der Auwera GA, et al. From FastQ data to high confidence variant calls: the Genome Analysis Toolkit best practices pipeline. Curr Protoc Bioinformatics. 2013;43:11.10.1-11.10.33.

50. Seelow D, Schuelke M, Hildebrandt F, Nürnberg P. HomozygosityMapper an interactive approach to homozygosity mapping. Nucleic Acids Res. 2009;37(Web Server issue):W593-W599.

51. Ahmad I, et al. Genetic heterogeneity in Pakistani microcephaly families revisited. Clin Genet. 2017;92(1):62-68.

52. MacArthur DG, et al. Guidelines for investigating causality of sequence variants in human disease. Nature. 2014;508(7497):469-476.

53. Moawia A, et al. Mutations of KIF14 cause primary microcephaly by impairing cytokinesis. Ann Neurol. 2017;82(4):562-577.

54. Szczepanski S, et al. A novel homozygous splicing mutation of CASC5 causes primary microcephaly in a large Pakistani family. Hum Genet. 2016;135(2):157-170.

55. Boehmer T, Schwartz TU. Purification, crystallization and preliminary X-ray analysis of a Nup107-Nup133 heterodimeric nucleoporin complex. Acta Crystallogr Sect F Struct Biol Cryst Commun. 2007;63(pt 9):816-818.

56. Saleem MA, et al. A conditionally immortalized human podocyte cell line demonstrating nephrin and podocin expression. J Am Soc Nephrol. 2002;13(3):630-638.

57. Hussain MS, et al. CDK6 associates with the centrosome during mitosis and is mutated in a large Pakistani family with primary microcephaly. Hum Mol Genet. 2013;22(25):5199-5214.

58. Hussain MS, et al. Mutations in CKAP2L, the human homolog of the mouse Radmis gene, cause Filippi syndrome. Am J Hum Genet. 2014;95(5):622-632.

59. Khokha MK, et al. Techniques and probes for the study of Xenopus tropicalis development. Dev Dyn. 2002;225(4):499-510.

60. Stoffel W, et al. Neutral sphingomyelinase (SMPD3) deficiency disrupts the Golgi secretory pathway and causes growth inhibition. Cell Death Dis. 2016;7(11):e2488.

61. Montague TG, Cruz JM, Gagnon JA, Church GM, Valen E. CHOPCHOP: a CRISPR/Cas9 and TALEN web tool for genome editing. Nucleic Acids Res. 2014;42(Web Server issue):W401-W407.

62. Vouillot L, Thélie A, Pollet N. Comparison of T7E1 and surveyor mismatch cleavage assays to detect mutations triggered by engineered nucleases. G3 (Bethesda). 2015;5(3):407-415. 